Article

\title{
Improved Time Response of Stabilization in Synchronization of Chaotic Oscillators Using Mathematica
}

\author{
Mohammad Shahzad ${ }^{1, *}$, Israr Ahmad ${ }^{1,2}$, Azizan Bin Saaban ${ }^{2}$ and Adyda Binti Ibrahim ${ }^{2}$ \\ 1 College of Applied Sciences, Ministry of Higher Education, Nizwa 611, Sultanate of Oman; \\ iak_2000plus@yahoo.com \\ 2 School of Quantitative Sciences, College of Arts \& Sciences, UUM, Keddha 06010, Malaysia; \\ azizan.s@uum.edu.my (A.B.S.); adyda@uum.edu.my (A.B.I.) \\ * Correspondence: dmsinfinite@gmail.com; Tel.: +968-9958-4197 \\ Academic Editor: Ockie Bosch
}

Received: 27 December 2015; Accepted: 15 June 2016; Published: 22 June 2016

\begin{abstract}
Chaotic dynamics are an interesting topic in nonlinear science that has been intensively studied during the last three decades due to its wide availability. Motivated by much researches on synchronization, the authors of this study have improved the time response of stabilization when parametrically excited $\Phi^{6}$-Van der Pol Oscillator (VDPO) and $\Phi^{6}$ —Duffing Oscillator (DO) are synchronized identically as well as non-identically (with each other) using the Linear Active Control (LAC) technique using Mathematica. Furthermore, the authors have synchronized the same pairs of the oscillators using a more robust synchronization with faster time response of stability called Robust Adaptive Sliding Mode Control (RASMC). A comparative study has been done between the previous results of Njah's work and our results based on Mathematica via LAC. The time response of stabilization of synchronization using RASMC has been discussed.
\end{abstract}

Keywords: chaos synchronization; LAC; Mathematica; chaotic oscillators; RASMC

\section{Introduction}

The study of chaotic behavior in nonlinear systems has attracted much attention because of many possible applications in various fields of science and technology. Most of the research has been devoted to the modeling of new chaotic systems together with the control and synchronization [1]. Thus far much work based on modeling, as well as various new control and synchronization techniques, has been carried out and is worth citing. For example, the sliding mode control [2-5], adaptive control [6-8], linear active control [9-13], linear feedback control [14-16], projective synchronization [17-19], nonlinear active control [20,21] and backstepping control [22], to mention but a few.

A recent study of Shahzad [23] focused the attention of researchers on how to choose a model based synchronization technique and the appropriate mathematical tools for simulation. Pourmahmood et al. [24] have developed a Robust Adaptive Sliding Mode Control (RASMC) and implemented it successfully on three well known chaotic systems (Lorenz, Chen and Liu) using MATLAB for all of the simulations, but when the same study was done using Mathematica by Shahzad [23], remarkable changes were observed in terms of time response to stabilize the synchronization.

Motivated by the aforementioned studies, we synchronize the identical and non-identical pairs of $\Phi^{6}-\mathrm{VDPO}$ and DO using the LAC and RASMC, respectively. However, the synchronization of $\Phi^{6}-\mathrm{VDPO}$ and DO using the LAC has already been done by Njah [12] but when the same work of Njah [12] was repeated via LAC and using Mathematica, remarkable changes had been found in the time response of stabilization of synchronization. On the other hand, the faster time 
response of stabilization of synchronization performances of the RASMC forced us to implement it on the same pairs of systems studied by Njah [12]. To the best of our knowledge, this kind of study has never been done before. The main objective behind the implementation of RASMC is that the sliding mode control is one of the robust control methods and has many interesting features such as low sensitivity to external disturbances and robustness to the plant uncertainties due to structural variations and un-modeled dynamics. The sliding mode controller is composed of an equivalent control part that describes the behavior of the system when the trajectories stay over the sliding surface and a variable structure control part that enforces the trajectories to reach the sliding surface and remain on it evermore. The adaptive control is a suitable approach to overcome system uncertainties, especially uncertainties derived from uncertain parameters. The adaptive sliding mode control has the advantages of combining the robustness of the sliding mode control with the tracking facilities of the adaptive control ([24], and the references therein).

The rest of the paper has been organized as follows: In Section 2, the three identical pairs of $\Phi^{6}-\mathrm{VDPO}$ and DO, respectively, and non-identical pairs of $\Phi^{6}-\mathrm{VDPO}$ and DO have been synchronized using the LAC. Section 3 is devoted to the brief description of RASMC as well as its implementation on identical synchronization of $\Phi^{6}-\mathrm{VDPO}$ and DO, respectively, and non-identical pair of $\Phi^{6}-$ VDPO and DO. Lastly, the whole study has been concluded in Section 4.

\section{Synchronization Using LAC}

In this section, we synchronize the identical and non-identical pairs of $\Phi^{6}-$ VDPO and DO, respectively, using the LAC technique for chaos synchronization that was proposed by Bai and Lonngren [9] and it has recently been accepted as one of the most efficient techniques for synchronizing both identical and non-identical chaotic systems because of its simple implementation in practical systems [25-28]. It can be easily designed according to the given conditions of the chaotic system as a way of accomplishing synchronization globally asymptotically, if the nonlinearity of the system is known. There are no derivatives in the controller and no need to calculate the Lyapunov exponents to execute the controller. These characteristics give an advantage to the technique over other conventional synchronization techniques.

\subsection{Description of the Models}

Since the chaotic systems are very complex nonlinear systems, they are exceptionally sensitive to tiny changes in their initial conditions and parameters variations. With the passage of time and due to the potential applications of chaotic systems in certain scientific fields, many chaotic and hyperchaotic systems have been investigated (Lorenz, Chen and Liu, etc.). In this direction, the $\Phi^{6}-\mathrm{VDPO}$ and DO are periodically self-excited and have rich applications in various disciplines like electronics, physics, engineering, neurology and biological sciences [29-31]. Njah [12] studied and investigated the synchronizations of $\Phi^{6}-\mathrm{VDPO}$ and $\mathrm{DO}$ with applications to secure communication using the LAC technique based on the Lyapunov stability theory and the Routh-Hurwitz criterion. The synchronization schemes have been studied without considering the external disturbances and model uncertainties. However, in practical applications, either environmental changes (noise) may occur any time or lack of parameters knowledge may disturb the stability of the synchronized system and this uncertainty or environmental noise cannot be simply ignored.

The $\Phi^{6}-$ VDPO and DO are classical examples of self-oscillatory and periodic systems and are now considered as very valuable mathematical models that can be utilized in much more complex and modified systems. In these models, there exist two frequencies, namely periodic forcing and self-oscillations. The energy is generated at low amplitudes and dissipated at high amplitude. The dynamics of chaotic parametrically excited $\Phi^{6}-\mathrm{VDPO}$ [12] are given by the following mathematical model:

$$
\Phi^{6}-\mathrm{VDPO}: \quad \ddot{x}-\mu_{1}\left(1-x^{2}\right) \dot{x}+\alpha_{1}\left\{1+\eta_{1} \cos \left(2 \omega_{1} t\right)\right\} x+\beta_{1} x^{3}+\lambda_{1} x^{5}=f_{1} \cos \omega_{1} t
$$


The dynamics of another chaotic parametrically excited $\Phi^{6}$-DO [12] are given by the following mathematical model:

$$
\Phi^{6}-\mathrm{DO}: \ddot{x}+\mu_{2} \dot{x}+\alpha_{2}\left\{1+\eta_{2} \cos \left(2 \omega_{2} t\right)\right\} x+\beta_{2} x^{3}+\lambda_{2} x^{5}=f_{2} \cos \omega_{2} t
$$

where the $\Phi^{6}-\mathrm{VDPO}$ and DO exhibit chaotic attractors for the following parameter values: $\mu_{1}=0.4$, $\alpha=1.0, \beta=-0.7, \lambda_{1}=0.1, \eta_{1}=0.7, f_{1}=9, \omega_{1}=3.14$ and $\mu_{2}=0.4, \alpha_{2}=0.46, \beta_{2}=1, \lambda_{2}=0.1$, $f_{2}=4.5, \eta_{2}=0.7, \omega_{2}=0.86$, respectively [12].

\subsection{Synchronization of Two Identical $\Phi^{6} — V D P O$ Oscillators via LAC}

To achieve synchronization between two identical $\Phi^{6}$ —VDPO, let us consider the master-slave systems synchronization scheme for two coupled identical chaotic $\Phi^{6}$-VDPO that can be written by choosing $x=x_{1}$ and $\dot{x}_{1}=x_{2}$ and $x=y_{1}$ and $\dot{x}=y_{2}$ in Equation (1) for master and slave systems, respectively, as follows:

$$
\begin{gathered}
\text { Master System : }\left\{\begin{array}{l}
\dot{x}_{1}=x_{2} \\
\dot{x}_{2}=\mu_{1}\left(1-x_{1}^{2}\right) x_{2}-\alpha_{1}\left\{1+\eta_{1} \cos \left(2 \omega_{1} t\right)\right\} x_{1}-\beta_{1} x_{1}^{3}-\lambda_{1} x_{1}^{5}+f_{1} \cos \omega_{1} t
\end{array}\right. \\
\text { Slave System : }\left\{\begin{array}{l}
\dot{y}_{1}=y_{2}+u_{1}(t), \\
\dot{y}_{2}=\mu_{1}\left(1-y_{1}^{2}\right) y_{2}-\alpha_{1}\left\{1+\eta_{1} \cos \left(2 \omega_{1} t\right)\right\} y_{1}-\beta_{1} y_{1}^{3}-\lambda_{1} y_{1}^{5}+f_{1} \cos \omega_{1} t+u_{2}(t)
\end{array}\right.
\end{gathered}
$$

where $\left[x_{1}(t), x_{2}(t)\right]^{T}$ and $\left[y_{1}(t), y_{2}(t)\right]^{T} \in R^{2}$ are the state variables of master and slave systems, respectively; $\mu_{1}, \alpha_{1}, \beta_{1}, \lambda_{1}, f_{1}, \eta_{1}$ and $\omega_{1}$ are the parameters involved in Equations (3) and (4) and $\boldsymbol{u}(t)=\left[u_{1}(t), u_{2}(t)\right]^{T} \in \boldsymbol{R}^{2 \times 1}$ are the control inputs yet to be determined.

Now, the error dynamics $\left(e_{i}=y_{i}-x_{i}\right.$, for $\left.i=1,2\right)$ from Equations (3) and (4) can be written as follows:

$$
\begin{aligned}
& \dot{e}_{1}=e_{2}+u_{1}(t) \\
& \dot{e}_{2}=\mu_{1} e_{1}-\mu_{1}\left(y_{1}^{2} y_{2}-x_{1}^{2} x_{2}\right)-\alpha_{1}\left\{1+\eta_{1} \cos \left(2 \omega_{1} t\right)\right\} e_{1}-\beta_{1}\left(y_{1}^{3}-x_{1}^{3}\right)-\lambda_{1}\left(y_{1}^{5}-x_{1}^{5}\right)+u_{2}(t)
\end{aligned}
$$

In order to make the error dynamics linear, let us redefine $u_{1} \& u_{2}$ as follows:

$$
\begin{aligned}
& u_{1}(t)=v_{1}(t) \\
& u_{2}(t)=\mu_{1}\left(y_{1}^{2} y_{2}-x_{1}^{2} x_{2}\right)+\beta_{1}\left(y_{1}^{3}-x_{1}^{3}\right)+\lambda_{1}\left(y_{1}^{5}-x_{1}^{5}\right)+v_{2}(t)
\end{aligned}
$$

$\therefore$ Now the linear error dynamical system can be written as:

$$
\begin{aligned}
& \dot{e}_{1}=e_{2}+v_{1}(t) \\
& \dot{e}_{2}=\mu_{1} e_{1}-\alpha_{1}\left\{1+\eta_{1} \cos \left(2 \omega_{1} t\right)\right\} e_{1}+v_{2}(t)
\end{aligned}
$$

The linear error dynamics (Equation (7)) is controlled by $v_{1}\left(e_{1}, e_{2}\right)$ and $v_{2}\left(e_{1}, e_{2}\right)$ that are defined as: $\left(\begin{array}{l}v_{1} \\ v_{2}\end{array}\right)=D\left(\begin{array}{l}e_{1} \\ e_{2}\end{array}\right)$ where $D=\left(\begin{array}{ll}a & b \\ c & d\end{array}\right)$ is a constant feedback matrix yet to be determined and the error dynamics (Equation (7)) can be written as: $\left(\begin{array}{c}\dot{e}_{1} \\ \dot{e}_{2}\end{array}\right)=C\left(\begin{array}{c}e_{1} \\ e_{2}\end{array}\right)$ where $C=\left(\begin{array}{cc}a & 1+b \\ c-\alpha_{1}\left\{1+\eta_{1} \cos \left(2 \omega_{1} t\right)\right\} & \mu_{1}+d\end{array}\right)$ is the coefficient matrix. According to the Lyapunov stability theory and Routh-Hurwitz criteria, choose $a+d+\mu_{1}<0$ and $\left\{c-\alpha_{1}\left(1+\eta_{1} \cos 2 \omega_{1} t\right)\right\}(1+b)-a\left(\mu_{1}+d\right)<0$ for the stabilization of the synchronization of Equations (3) and (4). 
Let $a+d+\mu_{1}=\left\{c-\alpha_{1}\left(1+\eta_{1} \cos 2 \omega_{1} t\right)\right\}(1+b)-a\left(\mu_{1}+d\right)=-E$ for $E>0$. We choose $a=b=0$ and $E=1$, which yields $u_{1}(t)=0$ and $u_{2}(t)=\mu_{1}\left(y_{1}^{2} y_{2}-x_{1}^{2} x_{2}\right)+\beta_{1}\left(y_{1}^{3}-x_{1}^{3}\right)+\lambda_{1}\left(y_{1}^{5}-\right.$ $\left.x_{1}^{5}\right)+\left\{\alpha_{1}\left(1+\eta_{1} \cos 2 \omega_{1} t\right)-E\right\} e_{1}-\left(\mu_{1}+E\right) e_{2}$.

For the same values of parameters $\left(\mu_{1}=0.4, \alpha_{1}=1, \beta_{1}=-0.7, \lambda_{1}=0.1, f_{1}=9, \eta_{1}=0.7\right.$, $\omega_{1}=3.14$ and $\left.E=1\right)$ and initial conditions $\left(x_{1}(0)=0.1 ; x_{2}(0)=0.2 ; y_{1}(0)=2.2 ; y_{2}(0)=0.05\right)$ taken by [12], we have repeated all simulations using Mathematica. The following are the graphs of synchronization of two identical $\Phi^{6}$-VDPO oscillators:

\subsection{Synchronization for Two Identical $\Phi^{6}-D O$ via $L A C$}

To achieve synchronization between two identical $\Phi^{6}-\mathrm{DO}$, let us consider the master-slave systems synchronization scheme for two coupled identical chaotic $\Phi^{6}$-DO that can be written by taking $x=x_{1}$ and $\dot{x}_{1}=x_{2}$ and $x=y_{1}$ and $\dot{x}=y_{2}$ in Equation (2) for master and slave systems, respectively, as follows:

$$
\text { Master System : }\left\{\begin{array}{l}
\dot{x}_{1}=x_{2} \\
\dot{x}_{2}=-\mu_{2} x_{2}-\alpha_{2}\left\{1+\eta_{2} \cos \left(2 \omega_{2} t\right)\right\} x_{1}-\beta_{2} x_{1}^{3}-\lambda_{2} x_{1}^{5}+f_{2} \cos \omega_{2} t
\end{array}\right.
$$

$$
\text { Slave System : }\left\{\begin{array}{l}
\dot{y}_{1}=y_{2}+u_{1}(t) \\
\dot{y}_{2}=-\mu_{2} y_{2}-\alpha_{2}\left\{1+\eta_{2} \cos \left(2 \omega_{2} t\right)\right\} y_{1}-\beta_{2} y_{1}^{3}-\lambda_{2} y_{1}^{5}+f_{2} \cos \omega_{2} t+u_{2}(t)
\end{array}\right.
$$

where $\left[x_{1}(t), x_{2}(t)\right]^{T}$ and $\left[y_{1}(t), y_{2}(t)\right]^{T} \in R^{2}$ are the state variables; $\mu_{2}, \alpha_{2}, \beta_{2}, \lambda_{2}, f_{2}, \eta_{2}$ and $\omega_{2}$ are the parameters involved in Equations (8) and (9); and $\boldsymbol{u}(t)=\left[u_{1}(t), u_{2}(t)\right]^{T} \in \boldsymbol{R}^{2 \times 1}$ are the control inputs yet to be determined.

Using LAC technique as in Section 2.2, someone can find the controllers $u_{1}(t)=0$ and $u_{2}(t)=\beta_{2}\left(y_{1}^{3}-x_{1}^{3}\right)+\lambda_{2}\left(y_{1}^{5}-x_{1}^{5}\right)+\left\{\alpha_{2}\left(1+\eta_{2} \cos 2 \omega_{2} t\right)-E\right\} e_{1}+\left(\mu_{2}-E\right) e_{2}$. For the same values of parameters $\left(\mu_{2}=0.4, \alpha_{2}=0.46, \beta_{2}=1, \lambda_{2}=0.1, f_{2}=4.5, \eta_{2}=0.7, \omega_{2}=0.86\right.$ and $\left.E=1\right)$ and initial conditions $\left(x_{1}(0)=0 ; x_{2}(0)=1.5 ; y_{1}(0)=0.5 ; y_{2}(0)=1\right)$ as taken by Njah [12], we have repeated all simulations using Mathematica. The following are the graphs of synchronization of two identical $\Phi^{6}-\mathrm{DO}$ :

\subsection{Synchronization for $\Phi^{6}-V D P O$ and $D O$ via $L A C$}

To achieve synchronization between $\Phi^{6}$ - VDPO and DO, let us consider the master-slave systems synchronization scheme for two coupled $\Phi^{6}-\mathrm{VDPO}$ and DO that can be written by taking $x=x_{1}$ and $\dot{x}_{1}=x_{2}$ and $x=y_{1}$ and $\dot{x}=y_{2}$ in Equations (1) and (2) for master and slave systems, respectively, as follows:

$$
\begin{aligned}
& \text { Master System : }\left\{\begin{array}{l}
\dot{x}_{1}=x_{2} \\
\dot{x}_{2}=\mu_{1}\left(1-x_{1}^{2}\right) x_{2}-\alpha_{1}\left\{1+\eta_{1} \cos \left(2 \omega_{1} t\right)\right\} x_{1}-\beta_{1} x_{1}^{3}-\lambda_{1} x_{1}^{5}+f_{1} \cos \omega_{1} t
\end{array}\right. \\
& \text { Slave System : }\left\{\begin{array}{l}
\dot{y}_{1}=y_{2}+u_{1}(t), \\
\dot{y}_{2}=-\mu_{2} y_{2}-\alpha_{2}\left\{1+\eta_{2} \cos \left(2 \omega_{2} t\right)\right\} y_{1}-\beta_{2} y_{1}^{3}-\lambda_{2} y_{1}^{5}+f_{2} \cos \omega_{2} t+u_{2}(t)
\end{array}\right.
\end{aligned}
$$

where $\left[x_{1}(t), x_{2}(t)\right]^{T}$ and $\left[y_{1}(t), y_{2}(t)\right]^{T} \in R^{2}$ are the state variables of master and slave systems; $\mu_{1}$, $\alpha_{1}, \beta_{1}, \lambda_{1}, f_{1}, \eta_{1}, \omega_{1}, \mu_{2}, \alpha_{2}, \beta_{2}, \lambda_{2}, f_{2}, \eta_{2}$ and $\omega_{2}$ are the parameters involved in Equations (10) and (11) and $\boldsymbol{u}(t)=\left[u_{1}(t), u_{2}(t)\right]^{T} \in \boldsymbol{R}^{2 \times 1}$ are the control inputs yet to be determined.

Using LAC technique, as it has been implemented in the last two subsections, someone can find the controllers:

$$
\begin{array}{ll}
u_{1}(t)=0 & \\
u_{2}(t)= & \left(\mu_{1}+\mu_{2}\right) x_{2}-\mu_{1} x_{1}^{2} x_{2}+\alpha_{2}\left(1+\eta_{2} \cos 2 \omega_{2} t\right) x_{1}-\alpha_{1}\left(1+\eta_{1} \cos 2 \omega_{1} t\right) x_{1}+\beta_{2} y_{1}^{3}-\beta_{1} x_{1}^{3} \\
& +\lambda_{2} y_{1}^{5}-\lambda_{1} x_{1}^{5}-f_{2} \cos \omega_{2} t+f_{1} \cos \omega_{1} t+\left\{\alpha_{2}\left(1+\eta_{2} \cos 2 \omega_{2} t\right)-E\right\} e_{1}+\left(\mu_{2}-E\right) e_{2}
\end{array}
$$


For the same values of parameters $\left(\mu_{1}=0.4, \alpha_{1}=1, \beta_{1}=-0.7, \lambda_{1}=0.1, f_{1}=9, \eta_{1}=0.7\right.$, $\omega_{1}=3.14, \mu_{2}=0.4, \alpha_{2}=0.46, \beta_{2}=1, \lambda_{2}=0.1, f_{2}=4.5, \eta_{2}=0.7, \omega_{2}=0.86$ and $\left.E=1\right)$ and initial conditions $\left(x_{1}(0)=0.1 ; x_{2}(0)=0.2 ; y_{1}(0)=0 ; y_{2}(0)=1.5\right)$ as taken by Njah [12], we have repeated all simulations using Mathematica. The following are the graphs of synchronization of $\Phi^{6}-$ VDPO and DO.

\subsection{Results and Discussions}

In Sections 2.2-2.4, three different pairs of $\Phi^{6}-$ VDPO and DO have been synchronized using LAC technique. In our study, all simulations are based on Mathematica that provide us the remarkable changes in the time of stabilization of synchronization. Earlier, in the same study of Njah [12], for identical pairs of $\Phi^{6}-\mathrm{VDPO}$ and non-identical pairs (i.e., $\Phi^{6}$-VDPO and DO), controllers were activated at around $t=60$ and for identical pairs of $\Phi^{6}-\mathrm{DO}$, the controllers were activated at around $t=100$. On the other hand, for the same pairs and same technique (LAC) if Mathematica is being used, someone can observe the remarkable changes in the time of stabilization (Figures 1-13). In our study, for all of the cases, not only do controllers activate around $t=4$ but secure communication scheme (Figures $3,4,7,8,11$ and 12), convergence of errors defined by $e(t)=\sqrt{e_{1}^{2}(t)+e_{2}^{2}(t)}$ (Figure 13) also start to stabilize at the same time when simulation is done using Mathematica. Furthermore, it may also be observed that the error states converged to the origin in the range of $[-0.5,1.5]$ very smoothly and quickly as compared to the work done by Njah [12]. These features give advantages to the current study.

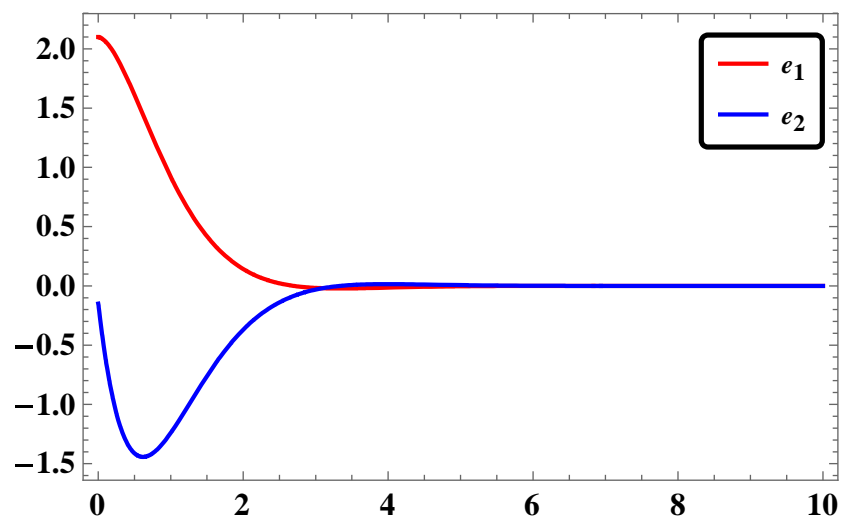

Figure 1. Time Series of $e_{1} \& e_{2}$.

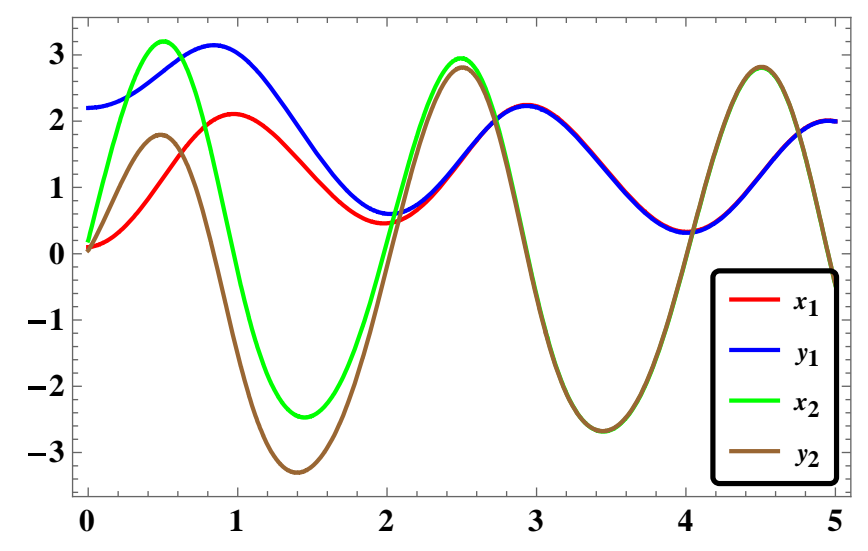

Figure 2. Time Series of $x_{1}, y_{1}, x_{2} \& y_{2}$. 


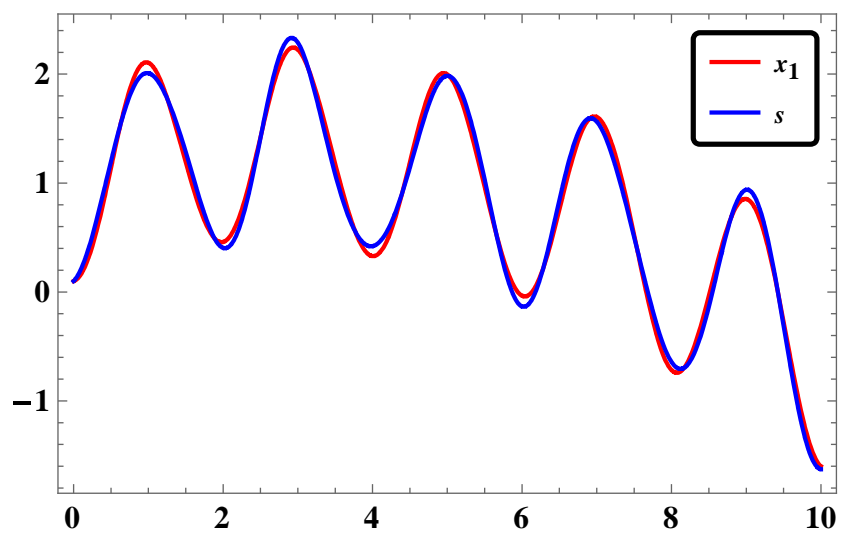

Figure 3. Time Series of $x_{1} \& s$.

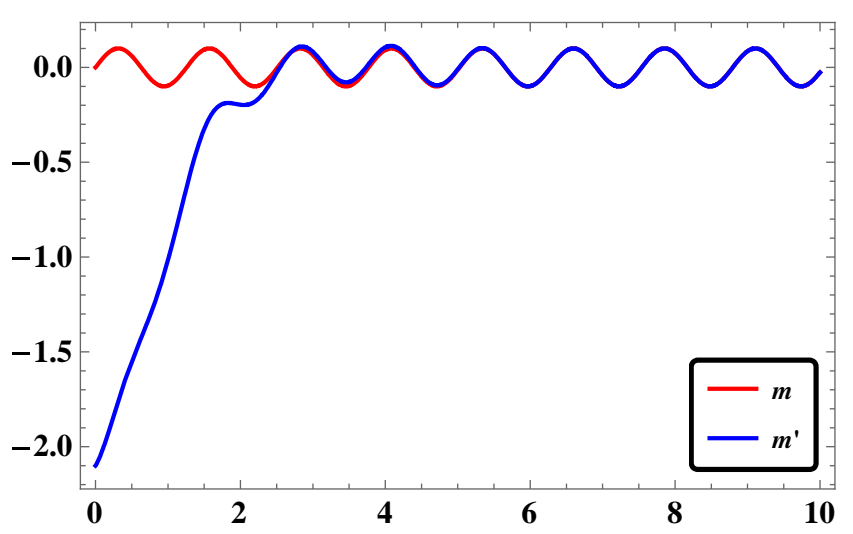

Figure 4. Time Series of $m \& m^{\prime}$.

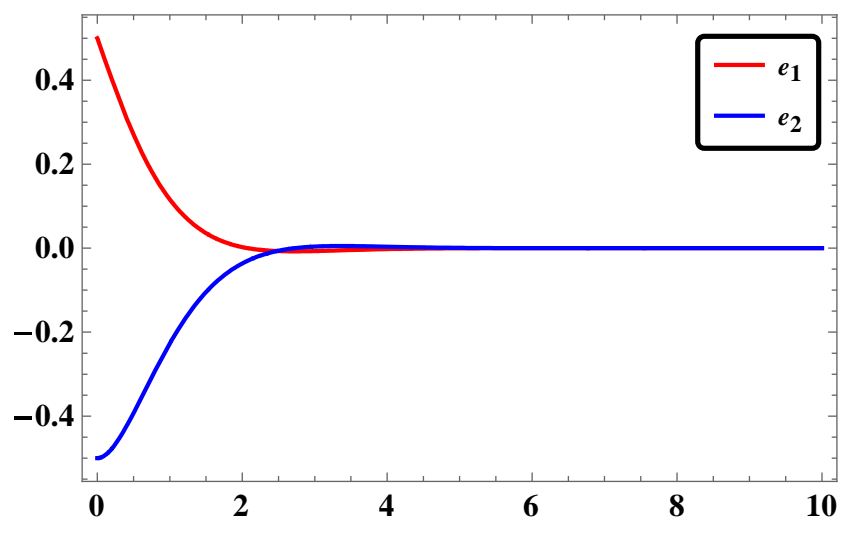

Figure 5. Time Series of $e_{1} \& e_{2}$. 


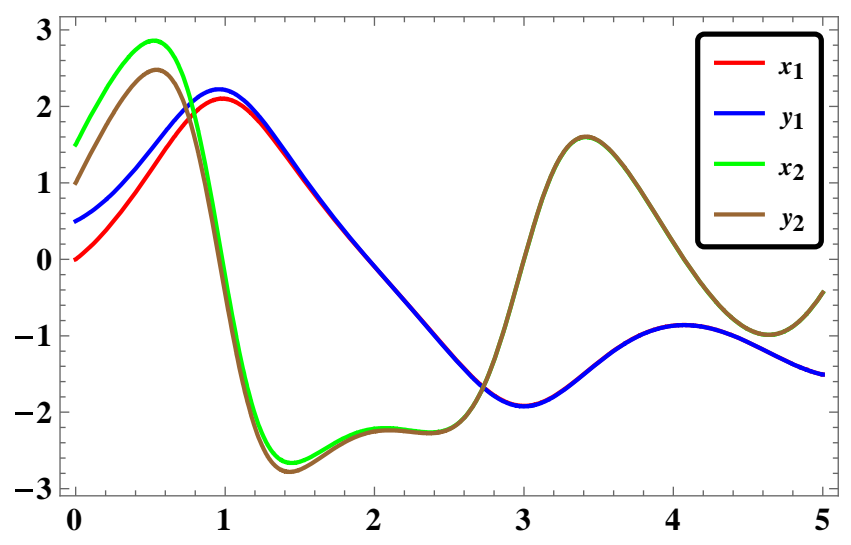

Figure 6. Time Series of $x_{1}, y_{1}, x_{2} \& y_{2}$.

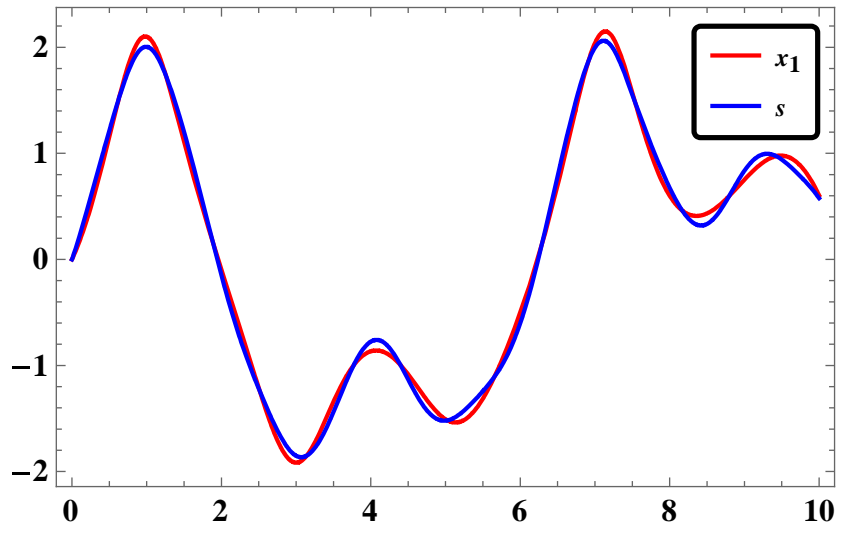

Figure 7. Time Series of $x_{1} \& s$.

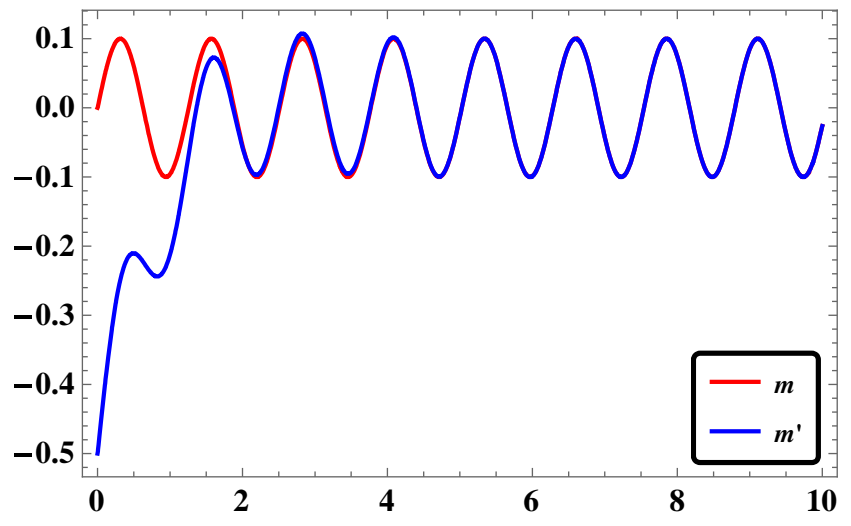

Figure 8. Time Series of $m \& m^{\prime}$. 


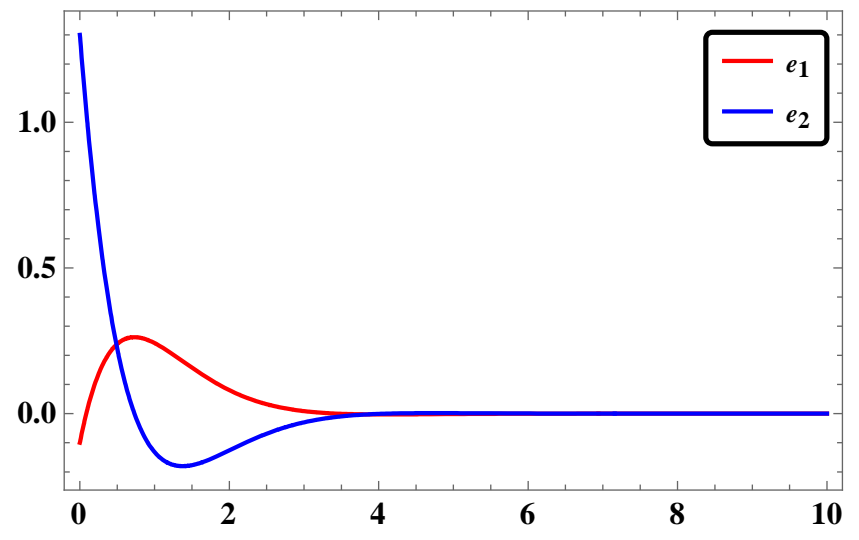

Figure 9. Time Series of $e_{1} \& e_{2}$.

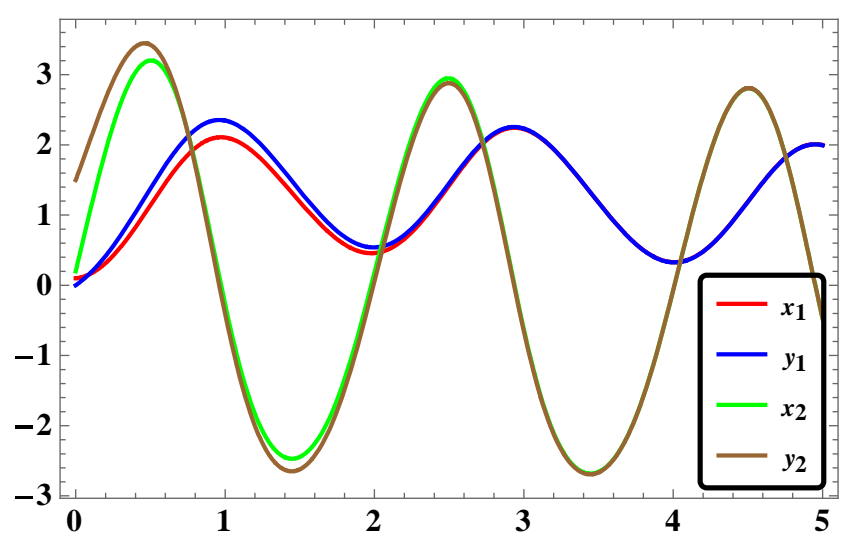

Figure 10. Time Series of $x_{1}, y_{1}, x_{2} \& y_{2}$.

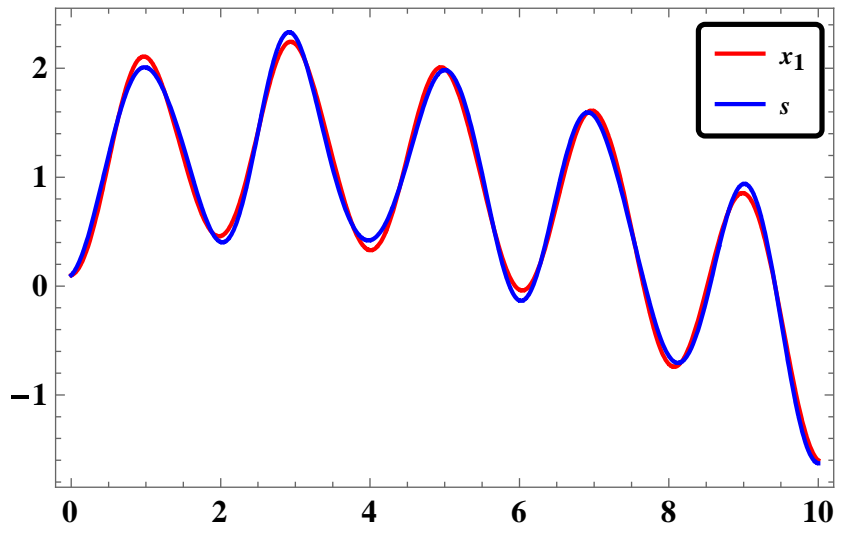

Figure 11. Time Series of $x_{1} \& s$. 


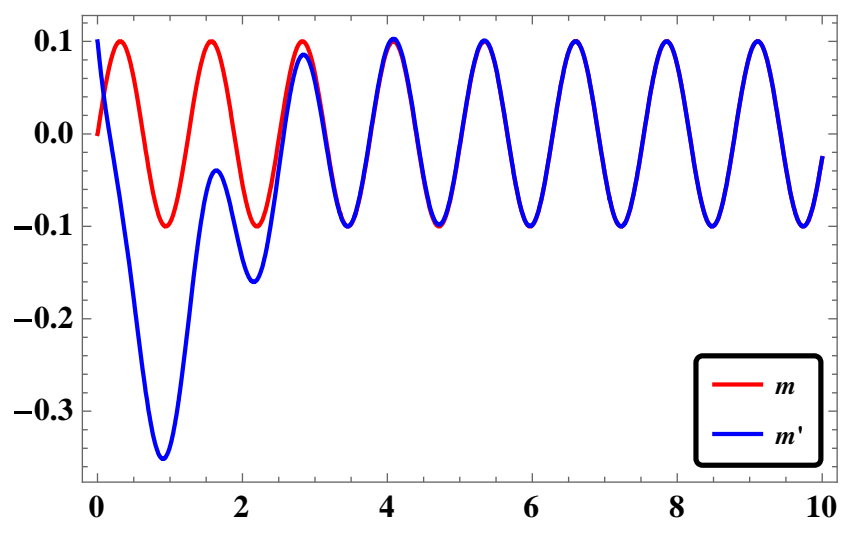

Figure 12. Time Series of $m \& m^{\prime}$.

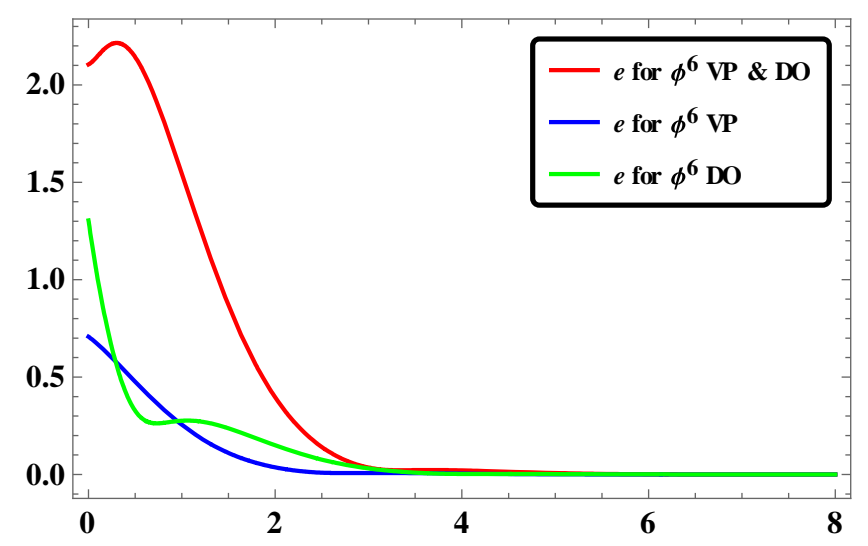

Figure 13. Time Series of $e$.

\section{Synchronization Using RASMC}

In this section, we synchronize the identical and non-identical pairs of $\Phi^{6}$-VDPO and DO using RASMC technique that has a very quick response in stabilizing the synchronization of chaotic systems [32-36]. Below we describe the technique in details.

\subsection{Description of RASMC}

For the $n$-dimensional master and slave systems with external uncertainties, disturbances and unknown parameters, the RASMC [24] is described as follows:

$$
\begin{gathered}
\text { Master system : } \dot{x}(t)=f(x)+\boldsymbol{F}(x) \boldsymbol{\theta}+\Delta f(x, t)+d^{m}(t) \\
\text { Slave System : } \dot{y}(t)=\boldsymbol{g}(y)+G(y) \psi+\Delta g(y, t)+d^{s}(t)+u(t)
\end{gathered}
$$

where $x(t)=\left[x_{1}, x_{2}, \ldots, x_{n}\right]^{T}$ are the state vectors, $f(x)=\left[f_{1}(x), f_{2}(x), \ldots, f_{n}(x)\right]^{T}$ are the continuous nonlinear functions, $F_{i}(\boldsymbol{x}), i=1,2, \ldots, n$, is $i^{t h}$ row of an $n \times n$ matrix $(\boldsymbol{F}(\boldsymbol{x}))$ whose elements are continuous nonlinear functions, $\boldsymbol{\theta}=\left[\theta_{1}, \theta_{2}, \ldots, \theta_{n}\right]^{T}$ are the unknown vector parameters, and $\Delta f(x, t)=\left[\Delta f_{1}(x, t), \Delta f_{2}(x, t), \ldots, \Delta f_{n}(x, t)\right]^{T}$ and $d^{m}(t)=\left[d_{1}^{m}(t), d_{2}^{m}(t), \ldots, d_{n}^{m}(t)\right]^{T}$ are the vectors of unknown uncertainties and external disturbances of the master system, respectively. $\boldsymbol{y}(t)=\left[y_{1}, y_{2}, \ldots, y_{n}\right]^{T}$ are the state vectors, $\boldsymbol{g}(\boldsymbol{y})=\left[g_{1}(\boldsymbol{y}), g_{2}(\boldsymbol{y}), \ldots, g_{n}(\boldsymbol{y})\right]^{T}$ are the continuous nonlinear functions, $G_{i}(\boldsymbol{y}), i=1,2, \ldots, n$, is $i^{t h}$ row of an $n \times n$ matrix $(\boldsymbol{G}(\boldsymbol{y}))$ whose elements are continuous nonlinear functions, $\psi=\left[\psi_{1}, \psi_{2}, \ldots, \psi_{n}\right]^{T}$ are the unknown vector parameters, $\Delta \boldsymbol{g}(\boldsymbol{y}, t)=\left[\Delta g_{1}(\boldsymbol{y}, t), \Delta g_{2}(\boldsymbol{y}, t), \ldots, \Delta g_{n}(\boldsymbol{y}, t)\right]^{T}$ and $\boldsymbol{d}^{s}(t)=\left[d_{1}^{s}(t), d_{2}^{s}(t), \ldots, d_{n}^{s}(t)\right]^{T}$ are the vectors 
of unknown uncertainties and external disturbances of the slave system, respectively, and $\boldsymbol{u}(t)=\left[u_{1}(t), u_{2}(t), \ldots, u_{n}(t)\right]^{T}$ is the vector of control inputs.

Assumption 1: Since the trajectories of chaotic systems are always bounded, then the unknown uncertainties $\Delta f(x, t)$ and $\Delta g(y, t)$ are assumed to be bounded. Therefore, there exist appropriate positive constants $\alpha_{i}^{m}$ and $\alpha_{i}^{s}, i=1,2, \ldots, n$ such that

$$
\begin{gathered}
\left|\Delta f_{i}(\boldsymbol{x}, t)\right|<\alpha_{i}^{m} \quad \text { and }\left|\Delta g_{i}(\boldsymbol{y}, t)\right|<\alpha_{i}^{s}, \quad i=1,2, \ldots n \\
\Rightarrow\left|\Delta f_{i}(\boldsymbol{x}, t)-\Delta g_{i}(\boldsymbol{y}, t)\right|<\alpha_{i}, \quad i=1,2, \ldots, n, \text { where } \alpha_{i} \text { are unknown constants }
\end{gathered}
$$

Assumption 2: In general, it is assumed that the external disturbances are norm-bounded in $C^{1}$,

$$
\begin{gathered}
\text { i.e., }\left|d_{i}^{m}(t)\right|<\beta_{i}^{m} \text { and }\left|d_{i}^{s}(t)\right|<\beta_{i}^{s}, \quad i=1,2, \ldots, n \\
\Rightarrow\left|d_{i}^{m}(t)-d_{i}^{s}(t)\right|<\beta_{i}, \quad i=1,2, \ldots, n \text {, where } \beta_{i} \text { are unknown constants }
\end{gathered}
$$

To solve the synchronization problem, the error between the master system (Equation (13)) and slave systems (Equation (14)) can be defined as $\boldsymbol{e}(t)=\boldsymbol{x}(t)-\boldsymbol{y}(t)$. Then, from Equations (13) and (14), the error dynamics can be written as:

$$
\dot{e}(t)=f(x)+F(x) \theta+\Delta f(x, t)+d^{m}(t)-g(y)-G(y) \psi-\Delta f(y, t)-d^{s}(t)-u(t)
$$

It is clear that the synchronization problem can be transformed to the equivalent problem of stabilizing the error system (Equation (19)). The objective of this paper is to show that for any given master chaotic system (Equation (13)) and slave chaotic system (Equation (14)) with the uncertainties, external disturbances and unknown parameters a suitable feedback control law $\boldsymbol{u}(t)$ is designed such that the asymptotical stability of the resulting error system (Equation (19)) can be achieved in the sense that $\lim _{t \rightarrow \infty}|x(t)-\boldsymbol{y}(t)| \rightarrow 0$ is for the systems under consideration.

Let us consider now the appropriate sliding surface with the desired behavior. Therefore, the sliding surface suitable for the technique can be designed as:

$$
s_{i}(t)=\lambda_{i} e_{i}(t), \quad i=1,2, \ldots, n
$$

where $s_{i}(t) \in \mathbb{R}\left(s(t)=\left[s_{1}(t), s_{2}(t), \ldots, s_{n}(t)\right]\right)$ and the sliding surface parameters $\lambda_{i}$ are positive constants.

After designing the suitable sliding surface, let us determine the input control signal $\boldsymbol{u}(t)$ to guarantee that the error system trajectories reach to the sliding surface $s(t)=0$ (i.e., to satisfy the reaching condition $\boldsymbol{s}(t) \dot{\boldsymbol{s}}(t)<0)$ and stay on it permanently. Therefore, to ensure the existence of the sliding motion a discontinuous control law (with minimum chattering effect) is proposed as:

$$
u_{i}(t)=f_{i}(\boldsymbol{x})-g_{i}(\boldsymbol{y})+F_{i}(\boldsymbol{x}) \hat{\theta}_{i}-G_{i}(\boldsymbol{y}) \hat{\psi}_{i}+\left(\hat{\alpha}_{i}+\hat{\beta}_{i}\right) \operatorname{sgn}\left(s_{i}\right)+k_{i} \tanh \left(\varepsilon s_{i}\right), \quad \text { for } i=1,2, \ldots, n
$$

where $\hat{\theta}_{i}, \hat{\psi}_{i}, \hat{\alpha}_{i}$, and $\hat{\beta}_{i}$ are estimations for $\theta_{i}, \psi_{i}$, and $\alpha_{i}$, respectively, $k_{i}>0, \quad i=1,2, \ldots, n$ are the switching gain constant, and $\varepsilon>0$.

To tackle the uncertainties, external disturbances and unknown parameters, appropriate update laws are defined as:

$$
\begin{aligned}
& \dot{\hat{\boldsymbol{\theta}}}=[\boldsymbol{F}(\boldsymbol{x})]^{T} \gamma, \quad \hat{\boldsymbol{\theta}}(0)=\hat{\boldsymbol{\theta}}_{0} \\
& \dot{\hat{\boldsymbol{\psi}}}=-[\boldsymbol{G}(\boldsymbol{y})]^{T} \gamma, \quad \hat{\boldsymbol{\psi}}(0)=\hat{\boldsymbol{\psi}}_{0} \\
& \dot{\hat{\alpha}}_{i}=\dot{\hat{\boldsymbol{\beta}}}_{i}=\lambda_{i}\left|s_{i}\right|, \quad \hat{\alpha}_{i}(0)=\hat{\alpha}_{i 0} \& \hat{\beta}_{i}(0)=\hat{\beta}_{i 0}
\end{aligned}
$$

where $\gamma=\left[\lambda_{1} s_{1}, \lambda_{2} s_{2}, \ldots, \lambda_{n} s_{n}\right]^{T}$ and $\hat{\boldsymbol{\theta}}_{0}, \hat{\psi}_{0}, \hat{\alpha}_{i 0}$ and $\hat{\beta}_{i 0}$ are the initial values of the update parameters $\hat{\boldsymbol{\theta}}, \hat{\psi}, \hat{\alpha}_{i}$ and $\hat{\beta}_{i}$, respectively. 
Based on the control input in Equation (21) and update laws in Equation (22) as used to guarantee the reaching condition $\boldsymbol{s}(t) \dot{\boldsymbol{s}}(t)<0$ and to ensure the occurrence of the sliding motion, we have the following theorem.

Theorem 1: Consider the error dynamics in Equation (19), this system is controlled by $\boldsymbol{u}(t)$ in Equation (13) with update laws in Equation (14). Then the error system trajectories will converge to the sliding surface $s(t)=0$.

In this regard, we consider a Lyapunov function (that is a positive definite function also) as follow:

$$
V(t)=\frac{1}{2} \sum_{i=1}^{n}\left[s_{i}^{2}+\left(\hat{\alpha}_{i}-\alpha_{i}\right)^{2}+\left(\hat{\beta}_{i}-\beta_{i}\right)^{2}\right]+\frac{1}{2}\|\hat{\boldsymbol{\theta}}-\boldsymbol{\theta}\|^{2}+\frac{1}{2}\|\hat{\boldsymbol{\psi}}-\boldsymbol{\psi}\|^{2}
$$

In order to apply the RASMC to synchronize the identical pairs of chaotic $\Phi^{6}-\mathrm{VDPO} ; \Phi^{6}-\mathrm{DO}$ and non-identical pair $\Phi^{6}-\mathrm{VDPO}$ and DO, external uncertainty for master and slave systems have been chosen as: $\Delta f_{i}\left(x_{i}, t\right) \rightarrow 0.5 \sin x_{i}$ and $\Delta g_{i}\left(y_{i}, t\right) \rightarrow-0.5 \sin y_{i}$, respectively; external disturbance for master and slave system: $d_{i}^{m}(t) \rightarrow 0.1 \sin t$ and $d_{i}^{s}(t) \rightarrow-0.1 \sin t$ for all $i=1,2$, respectively; $\varepsilon=100$; initial values of update parameters $\hat{\theta}_{i}(0)=1, \hat{\psi}_{i}(0)=2, \hat{\alpha}_{i}(0)=3$ and $\hat{\beta}_{i}(0)=4$; and for secure communication message signal $(m)=0.05 \sin 2 t$.

\subsection{Synchronization of Two Identical $\Phi^{6}-V D P O$ Using RASMC}

In this section, we synchronize the identical pairs of chaotic $\Phi^{6}$ - VDPO using RASMC under the effect of external uncertainty and external disturbance for both master and slave systems. After adding the external uncertainty and disturbances, Equation (1) can be written as a pair of master and slave systems:

$$
\begin{gathered}
\dot{\boldsymbol{x}}=\underbrace{\left[\begin{array}{c}
0 \\
A
\end{array}\right]}_{f(x)}+\underbrace{\left[\begin{array}{cc}
x_{2} & 0 \\
0 & x_{2}
\end{array}\right]}_{\boldsymbol{F}(\boldsymbol{x}, t)} \underbrace{\left[\begin{array}{c}
1 \\
\mu_{1}
\end{array}\right]}_{\boldsymbol{\theta}}+\underbrace{\left[\begin{array}{c}
0.5 \sin x_{1} \\
0.5 \sin x_{2}
\end{array}\right]}_{\Delta f(x, t)}+\underbrace{\left[\begin{array}{c}
0.1 \sin t \\
0.1 \sin t
\end{array}\right]}_{\boldsymbol{d}^{m}(t)} \\
\dot{\boldsymbol{y}}=\underbrace{\left[\begin{array}{c}
0 \\
B
\end{array}\right]}_{\boldsymbol{g}(\boldsymbol{y})}+\underbrace{\left[\begin{array}{cc}
y_{2} & 0 \\
0 & y_{2}
\end{array}\right]}_{\boldsymbol{G}(\boldsymbol{y}, t)} \underbrace{\left[\begin{array}{c}
1 \\
\mu_{1}
\end{array}\right]}_{\boldsymbol{\psi}}+\underbrace{\left[\begin{array}{l}
-0.5 \sin y_{1} \\
-0.5 \sin y_{2}
\end{array}\right]}_{\Delta \boldsymbol{g}(\boldsymbol{y}, t)}+\underbrace{\left[\begin{array}{l}
-0.1 \sin t \\
-0.1 \sin t
\end{array}\right]}_{\boldsymbol{d}^{m}(t)}+\underbrace{\left[\begin{array}{l}
u_{1}(t) \\
u_{2}(t)
\end{array}\right]}_{\boldsymbol{u}(t)}
\end{gathered}
$$

where $A=-\mu_{1} x_{1}^{2} x_{2}-\alpha_{1}\left(1+\eta_{1} \cos 2 \omega_{1} t\right) x_{1}-\beta_{1} x_{1}^{3}-\lambda_{1} x_{1}^{5}+f_{1} \cos \omega_{1} t, \quad B=-\mu_{1} y_{1}^{2} y_{2}-$ $\alpha_{1}\left(1+\eta_{1} \cos 2 \omega_{1} t\right) y_{1}-\beta_{1} y_{1}^{3}-\lambda_{1} y_{1}^{5}+f_{1} \cos \omega_{1} t$ and $u_{i}(t)$ for $i=1,2$ are the controllers which govern as per the rule (Equation (21)). Furthermore, during simulation, the initial values of states vectors in master and slave systems are chosen as: $x_{1}(0)=0.1, x_{2}(0)=0.2, y_{1}(0)=2.2, y_{2}(0)=0.05$, respectively; sliding surface parameters: $\lambda_{1}=25, \lambda_{2}=10$ and switching gain constants: $k_{1}=1, k_{2}=5$.

Therefore, using Equation (19), the error dynamics can be expressed as:

$$
\begin{aligned}
\dot{e}_{1}= & e_{2}+0.5\left(\sin x_{1}+\sin y_{1}\right)+0.2 \sin t-u_{1}(t), \\
\dot{e}_{2}= & \mu_{1} e_{1}-\mu_{1}\left(x_{1}^{2} x_{2}+y_{1}^{2} y_{2}\right)-\alpha_{1}\left\{1+\eta_{1} \cos \left(2 \omega_{1} t\right)\right\} e_{1}-\beta_{1}\left(x_{1}^{3}-y_{1}^{3}\right) \\
& -\lambda_{1}\left(x_{1}^{5}-y_{1}^{5}\right)+0.5\left(\sin x_{2}+\sin y_{2}\right)+0.2 \sin t-u_{2}(t) .
\end{aligned}
$$

where $u_{i}(t)=f_{i}(x)-g_{i}(y)+F_{i}(x) \hat{\theta}_{i}-G_{i}(y) \hat{\psi}_{i}+\left(\hat{\alpha}_{i}+\hat{\beta}_{i}\right) \operatorname{sign}\left(s_{i}\right)+k_{i} \tanh \left(\varepsilon s_{i}\right)$ for $i=1,2$ and the unknown parameters have been taken as per Equation (22). The following are the time series of synchronization errors (Figure 14), update parameters (Figures 15 and 16), states vectors (Figure 17) and for secure communication scheme (Figures 18 and 19). 


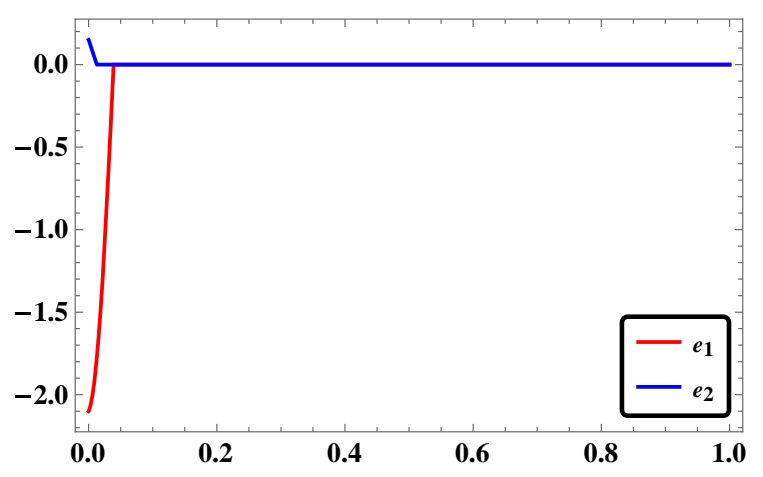

Figure 14. Time Series of $e_{1} \& e_{2}$.

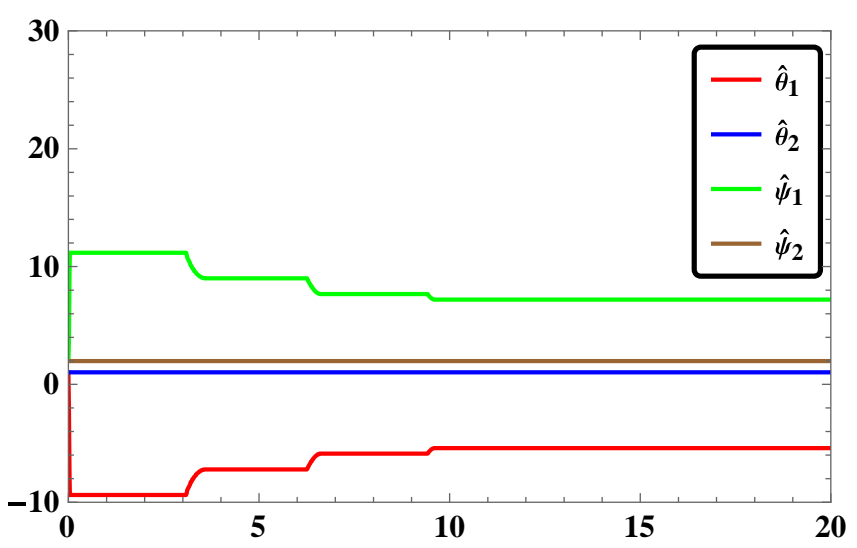

Figure 15. Time Series of $\hat{\theta}_{1}, \hat{\theta}_{2}, \hat{\psi}_{1} \& \hat{\psi}_{2}$.

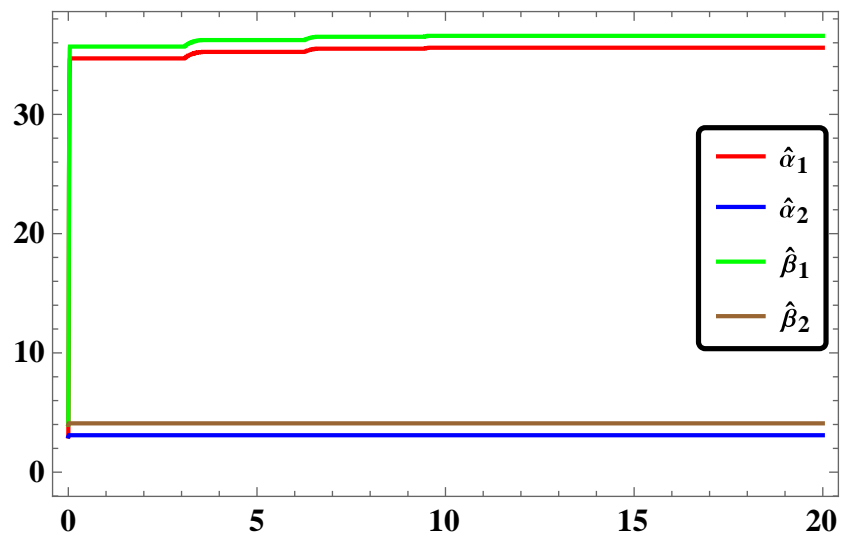

Figure 16. Time Series of $\hat{\alpha}_{1}, \hat{\alpha}_{2}, \hat{\beta}_{1} \& \hat{\beta}_{2}$. 


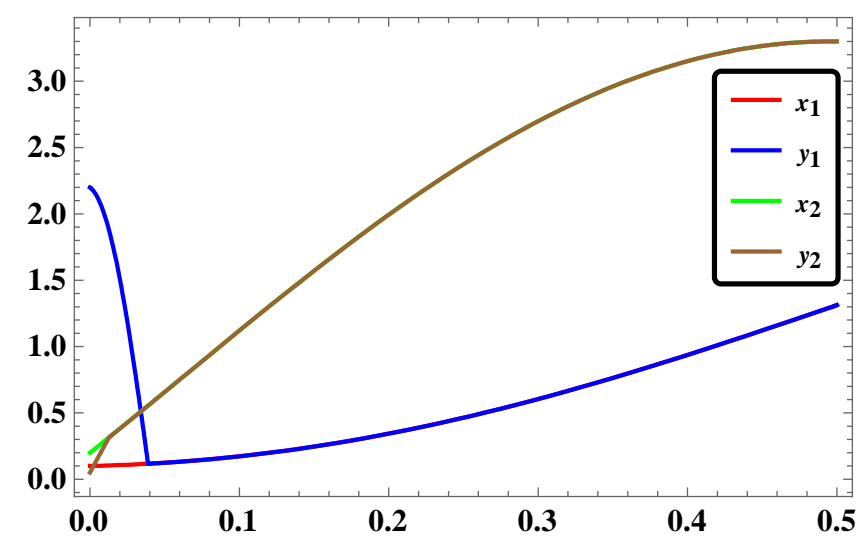

Figure 17. Time Series of $x_{1}, y_{1}, x_{2} \& y_{2}$.

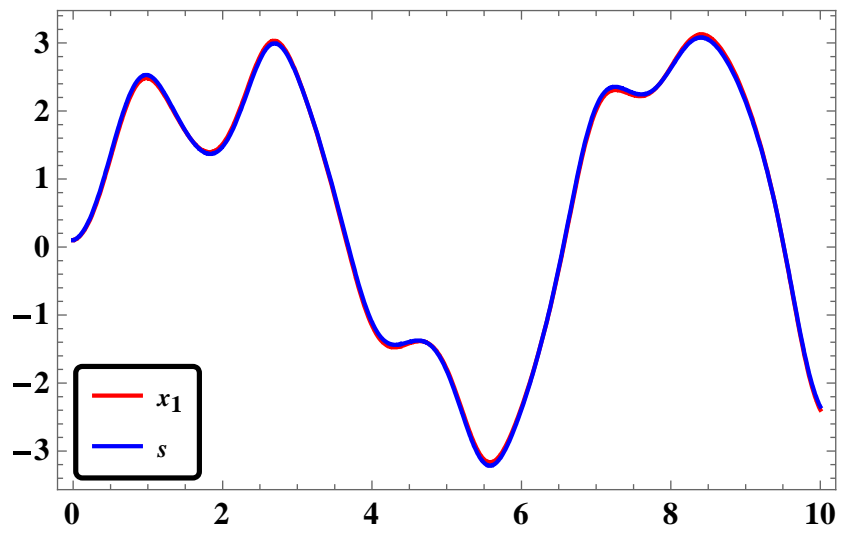

Figure 18. Time Series of $x_{1} \& s$.

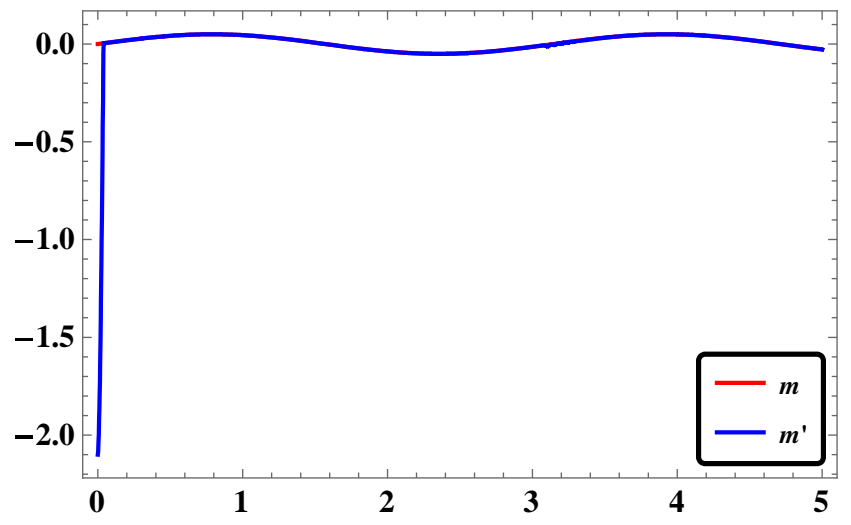

Figure 19. Time Series of $m \& m^{\prime}$.

\subsection{Synchronization of Two Identical $\Phi^{6}$-DO Using RASMC}

In this section, we synchronize the identical pairs of chaotic $\Phi^{6}-$ DO using RASMC under the effect of external uncertainty and external disturbance for both master and slave systems. After adding the external uncertainty and disturbances, Equation (2) can be written as a pair of master and slave systems:

$$
\dot{x}=\underbrace{\left[\begin{array}{c}
0 \\
A
\end{array}\right]}_{f(x)}+\underbrace{\left[\begin{array}{cc}
x_{2} & 0 \\
0 & -x_{2}
\end{array}\right]}_{\boldsymbol{F}(\boldsymbol{x}, t)} \underbrace{\left[\begin{array}{c}
1 \\
\mu_{2}
\end{array}\right]}_{\boldsymbol{\theta}}+\underbrace{\left[\begin{array}{c}
0.5 \sin x_{1} \\
0.5 \sin x_{2}
\end{array}\right]}_{\Delta f(x, t)}+\underbrace{\left[\begin{array}{c}
0.1 \sin t \\
0.1 \sin t
\end{array}\right]}_{\boldsymbol{d}^{m}(t)}
$$




$$
\dot{y}=\underbrace{\left[\begin{array}{l}
0 \\
B
\end{array}\right]}_{g(y)}+\underbrace{\left[\begin{array}{cc}
y_{2} & 0 \\
0 & -y_{2}
\end{array}\right]}_{\boldsymbol{G}(\boldsymbol{y}, t)} \underbrace{\left[\begin{array}{c}
1 \\
\mu_{2}
\end{array}\right]}_{\boldsymbol{\psi}}+\underbrace{\left[\begin{array}{l}
-0.5 \sin y_{1} \\
-0.5 \sin y_{2}
\end{array}\right]}_{\Delta \boldsymbol{g}(\boldsymbol{y}, t)}+\underbrace{\left[\begin{array}{c}
-0.1 \sin t \\
-0.1 \sin t
\end{array}\right]}_{\boldsymbol{d}^{m}(t)}+\underbrace{\left[\begin{array}{l}
u_{1}(t) \\
u_{2}(t)
\end{array}\right]}_{\boldsymbol{u}(t)}
$$

where

$$
\begin{aligned}
& A=-\alpha_{2}\left\{1+\eta_{2} \cos \left(2 \omega_{2} t\right)\right\} x_{1}-\beta_{2} x_{1}^{3}-\lambda_{2} x_{1}^{5}+f_{2} \cos \omega_{2} t, \\
& B=-\alpha_{2}\left\{1+\eta_{2} \cos \left(2 \omega_{2} t\right)\right\} y_{1}-\beta_{2} y_{1}^{3}-\lambda_{2} y_{1}^{5}+f_{2} \cos \omega_{2} t
\end{aligned}
$$

and $u_{i}(t)$ for $i=1,2$ are the controllers which govern as per the rule (Equation (22)). Furthermore, during simulation, the initial values of states vectors in master and slave systems are chosen as: $x_{1}(0)=0, x_{2}(0)=1.5, y_{1}(0)=0.5, y_{2}(0)=1$, respectively; sliding surface parameters: $\lambda_{1}=12, \lambda_{2}=10$; and switching gain constants: $k_{1}=k_{2}=20$.

Therefore, using Equation (19), the error dynamics can be expressed as:

$$
\begin{aligned}
\dot{e}_{1}= & e_{2}+0.5\left(\sin x_{1}+\sin y_{1}\right)+0.2 \sin t-u_{1}(t), \\
\dot{e}_{2}= & -\mu_{2} e_{1}-\alpha_{2}\left\{1+\eta_{1} \cos \left(2 \omega_{1} t\right)\right\} e_{1}-\beta_{1}\left(x_{1}^{3}-y_{1}^{3}\right)-\lambda_{1}\left(x_{1}^{5}-y_{1}^{5}\right) \\
& +0.5\left(\sin x_{2}+\sin y_{2}\right)+0.2 \sin t-u_{2}(t) .
\end{aligned}
$$

where $u_{i}(t)=f_{i}(x)-g_{i}(y)+F_{i}(x) \hat{\theta}_{i}-G_{i}(y) \hat{\psi}_{i}+\left(\hat{\alpha}_{i}+\hat{\beta}_{i}\right) \operatorname{sign}\left(s_{i}\right)+k_{i} \tanh \left(\varepsilon s_{i}\right)$ for $i=1,2$ and the unknown parameters have been taken as per (22). The following are the time series of synchronization errors (Figure 20), update parameters (Figures 21 and 22), states vectors (Figure 23) and for secure communication scheme (Figures 24 and 25).

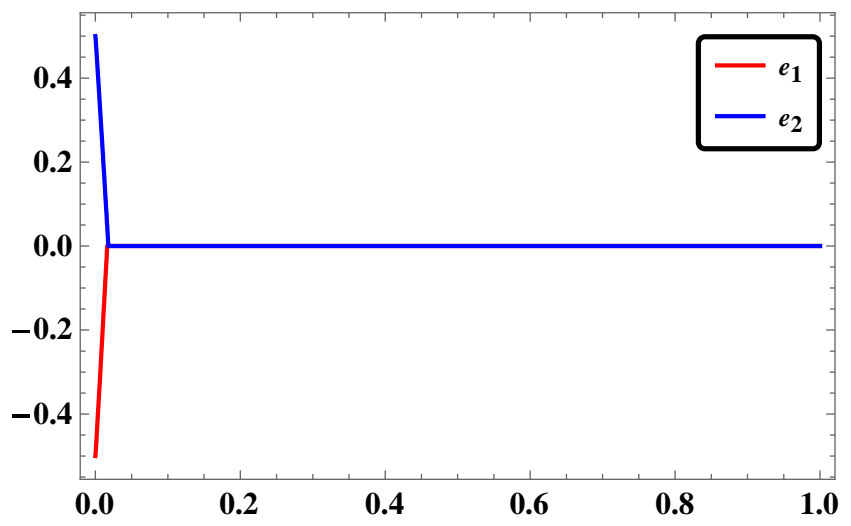

Figure 20. Time Series of $e_{1} \& e_{2}$.

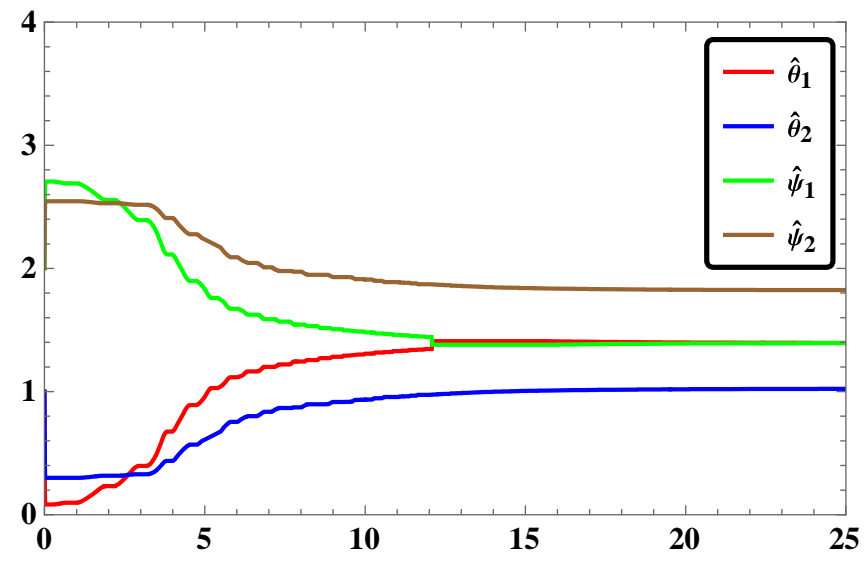

Figure 21. Time Series of $\hat{\theta}_{1}, \hat{\theta}_{2}, \hat{\psi}_{1} \& \hat{\psi}_{2}$. 


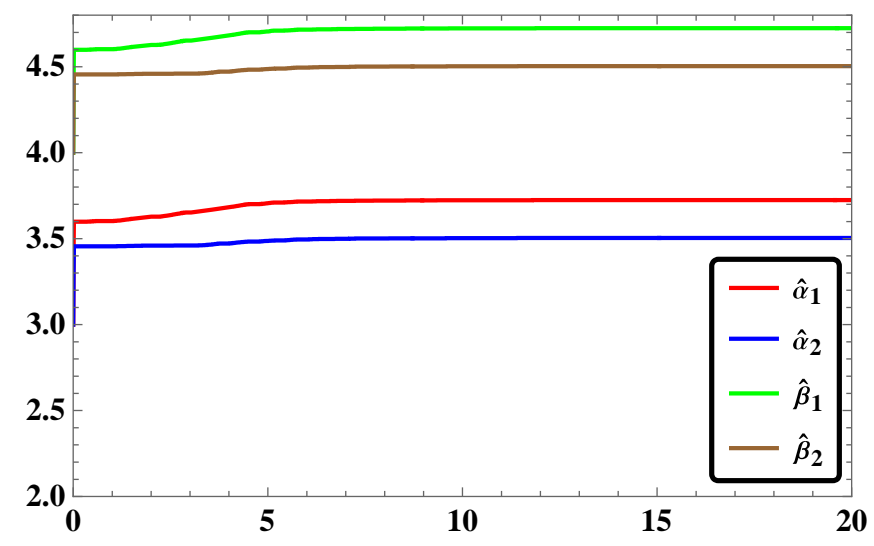

Figure 22. Time Series of $\hat{\alpha}_{1}, \hat{\alpha}_{2}, \hat{\beta}_{1} \& \hat{\beta}_{2}$.

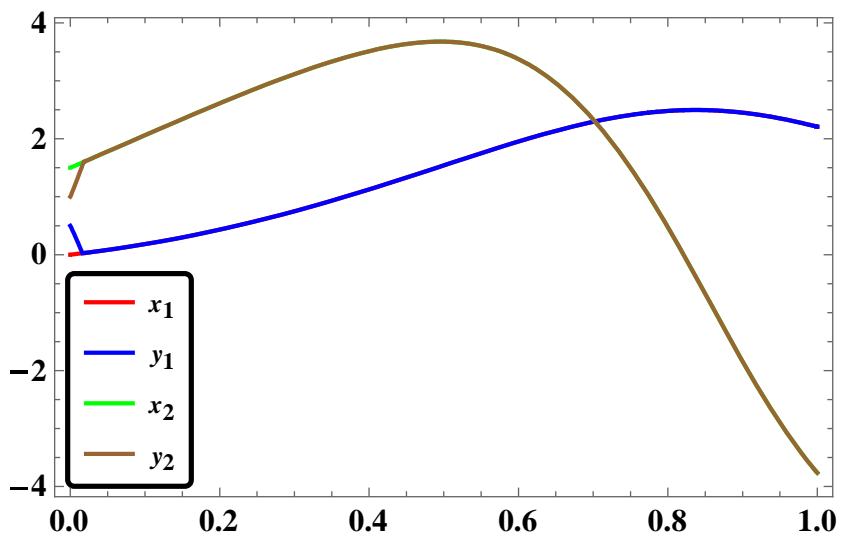

Figure 23. Time Series of $x_{1}, y_{1}, x_{2} \& y_{2}$.

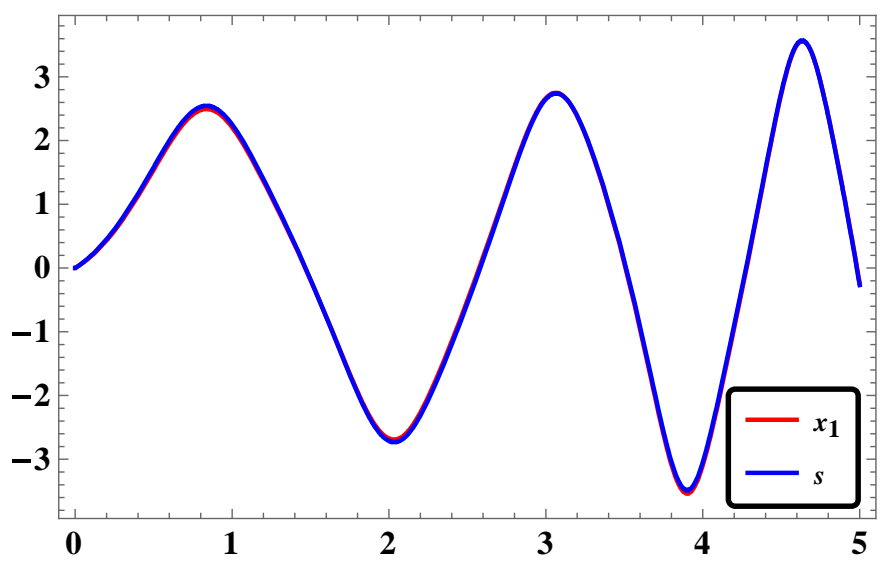

Figure 24. Time Series of $x_{1} \& s$. 


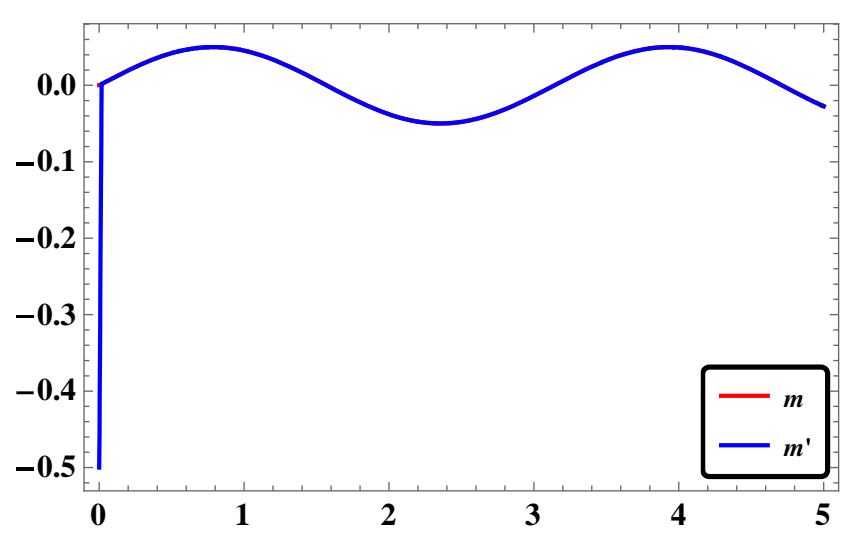

Figure 25. Time Series of $m \& m^{\prime}$.

\subsection{Synchronization of $\Phi^{6}-V D P O$ and $D O U$ using $R A S M C$}

In this section, we synchronize the non-identical pairs of chaotic $\Phi^{6}-\mathrm{VDPO}$ and DO using RASMC under the effect of external uncertainty and external disturbance for both master and slave systems. After adding the external uncertainty and disturbances, Equations (1) and (2) can be written as a pair of master and slave systems, respectively:

$$
\begin{gathered}
\dot{x}=\underbrace{\left[\begin{array}{c}
0 \\
A
\end{array}\right]}_{f(x)}+\underbrace{\left[\begin{array}{cc}
x_{2} & 0 \\
0 & x_{2}
\end{array}\right]}_{\boldsymbol{F}(\boldsymbol{x}, t)} \underbrace{\left[\begin{array}{c}
1 \\
\mu_{1}
\end{array}\right]}_{\boldsymbol{\theta}}+\underbrace{\left[\begin{array}{l}
0.5 \sin x_{1} \\
0.5 \sin x_{2}
\end{array}\right]}_{\Delta f(x, t)}+\underbrace{\left[\begin{array}{c}
0.1 \sin t \\
0.1 \sin t
\end{array}\right]}_{\boldsymbol{d}^{m}(t)} \\
\dot{y}=\underbrace{\left[\begin{array}{c}
0 \\
B
\end{array}\right]}_{\boldsymbol{g}(\boldsymbol{y})}+\underbrace{\left[\begin{array}{cc}
y_{2} & 0 \\
0 & -y_{2}
\end{array}\right]}_{\boldsymbol{G}(\boldsymbol{y}, t)} \underbrace{\left[\begin{array}{c}
1 \\
\mu_{2}
\end{array}\right]}_{\boldsymbol{\psi}}+\underbrace{\left[\begin{array}{l}
-0.5 \sin y_{1} \\
-0.5 \sin y_{2}
\end{array}\right]}_{\Delta \boldsymbol{g}(\boldsymbol{y}, t)}+\underbrace{\left[\begin{array}{l}
-0.1 \sin t \\
-0.1 \sin t
\end{array}\right]}_{\boldsymbol{d}^{m}(t)}+\underbrace{\left[\begin{array}{l}
u_{1}(t) \\
u_{2}(t)
\end{array}\right]}_{\boldsymbol{u}(t)}
\end{gathered}
$$

where

$$
\begin{gathered}
A=-\mu_{1} x_{1}^{2} x_{2}-\alpha_{1}\left\{1+\eta_{1} \cos \left(2 \omega_{1} t\right)\right\} x_{1}-\beta_{1} x_{1}^{3}-\lambda_{1} x_{1}^{5}+f_{1} \cos \omega_{1} t \\
B=-\alpha_{2}\left\{1+\eta_{2} \cos \left(2 \omega_{2} t\right)\right\} y_{1}-\beta_{2} y_{1}^{3}-\lambda_{2} y_{1}^{5}+f_{2} \cos \omega_{2} t
\end{gathered}
$$

and $u_{i}(t)$ for $i=1,2$ are the controllers which govern as per the rule (Equation (22)). Furthermore, during simulation, the initial values of states vectors in master and slave systems are chosen as: $x_{1}(0)=0.1, x_{2}(0)=0.2, y_{1}(0)=0$, and $y_{2}(0)=1.5$, respectively; sliding surface parameters: $\lambda_{1}=12$, $\lambda_{2}=10$; and switching gain constants: $k_{1}=k_{2}=20$.

Therefore, using Equation (19), the error dynamics can be expressed as:

$$
\begin{aligned}
\dot{e}_{1}= & e_{2}+0.5\left(\sin x_{1}+\sin y_{1}\right)+0.2 \sin t-u_{1}(t) \\
\dot{e}_{2}= & \mu_{1}\left(1-x_{1}^{2}\right) x_{2}-\alpha_{1}\left\{1+\eta_{1} \cos \left(2 \omega_{1} t\right)\right\} x_{1}-\beta_{1} x_{1}^{3}+\beta_{2} y_{1}^{3}-\lambda_{1} x_{1}^{5}+\lambda_{2} y_{1}^{5}+f_{1} \cos \omega_{1} t \\
& +\mu_{2} y_{2}+\alpha_{2}\left\{1+\eta_{1} \cos \left(2 \omega_{2} t\right)\right\} y_{1}+f_{2} \cos \omega_{2} t+0.5\left(\sin x_{2}+\sin y_{2}\right)+0.2 \sin t-u_{2}(t) .
\end{aligned}
$$

where $u_{i}(t)=f_{i}(x)-g_{i}(y)+F_{i}(x) \hat{\theta}_{i}-G_{i}(y) \hat{\psi}_{i}+\left(\hat{\alpha}_{i}+\hat{\beta}_{i}\right) \operatorname{sign}\left(s_{i}\right)+k_{i} \tanh \left(\varepsilon s_{i}\right)$ for $i=1,2$ and the unknown parameters have been taken as per Equation (22). The following are the time series of synchronization errors (Figure 26), update parameters (Figures 27 and 28), states vectors (Figure 29) and for secure communication scheme (Figures 30 and 31) as well as the derivative of Lyapunov function $(\dot{V}(t))$ for all three pairs together (Figure 32). 


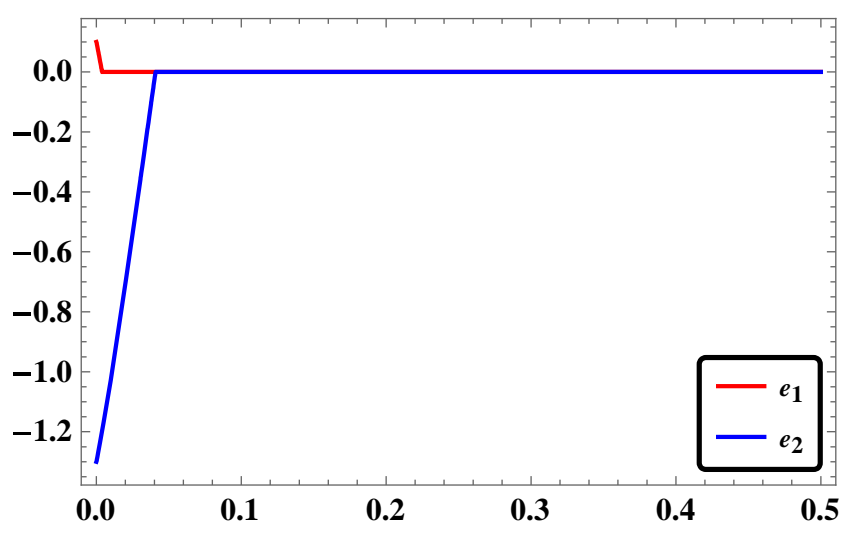

Figure 26. Time Series of $e_{1} \& e_{2}$.

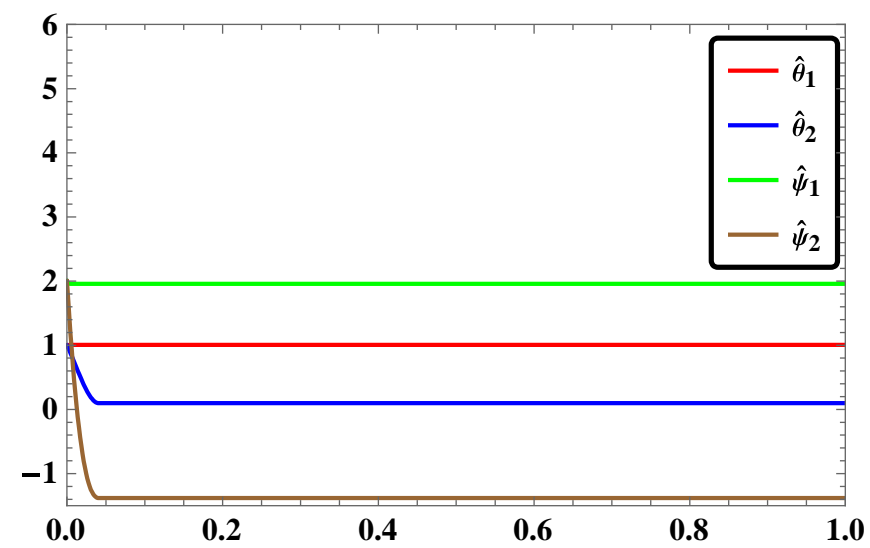

Figure 27. Time Series of $\hat{\theta}_{1}, \hat{\theta}_{2}, \hat{\psi}_{1} \& \hat{\psi}_{2}$.

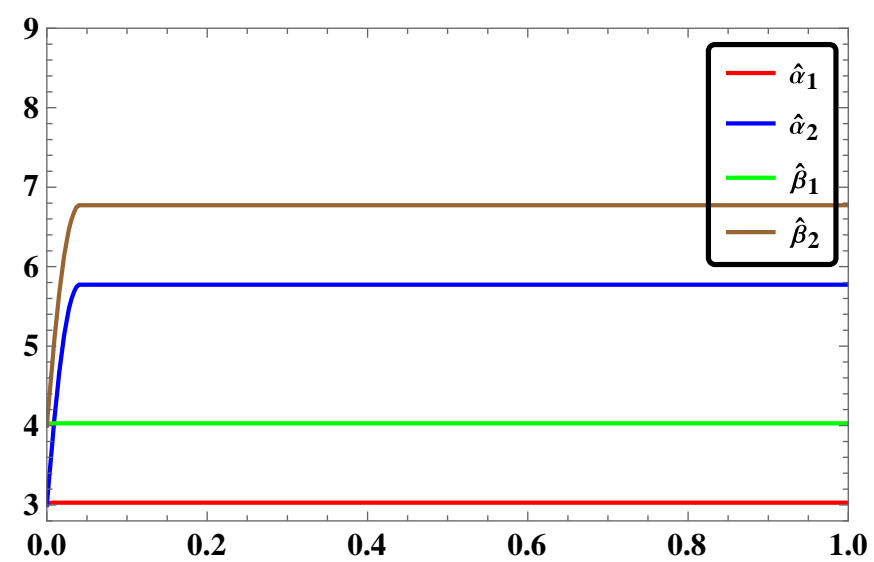

Figure 28. Time Series of $\hat{\alpha}_{1}, \hat{\alpha}_{2}, \hat{\beta}_{1} \& \hat{\beta}_{2}$. 


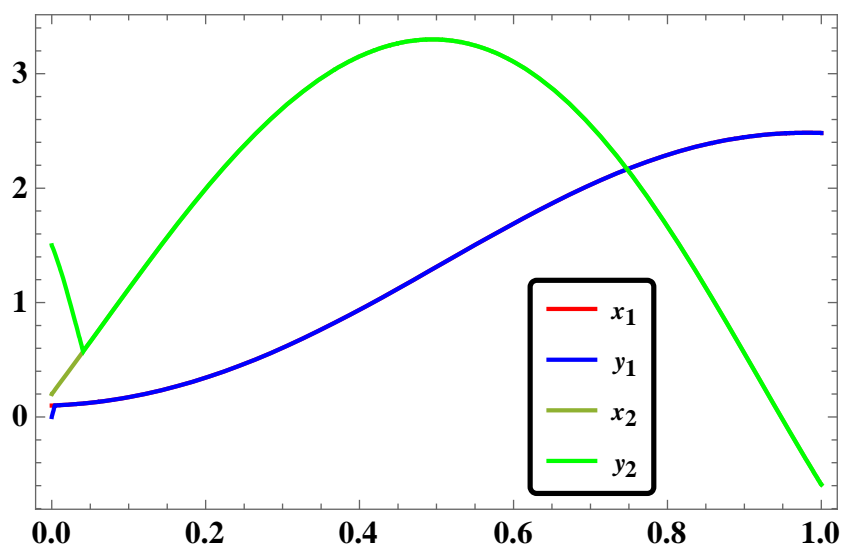

Figure 29. Time Series of $x_{1}, y_{1}, x_{2} \& y_{2}$.

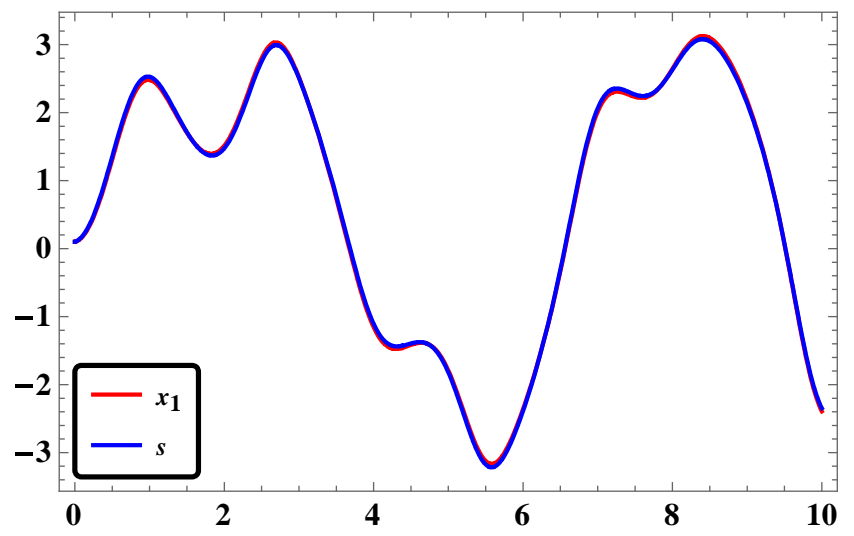

Figure 30. Time Series of $x_{1} \& s$.

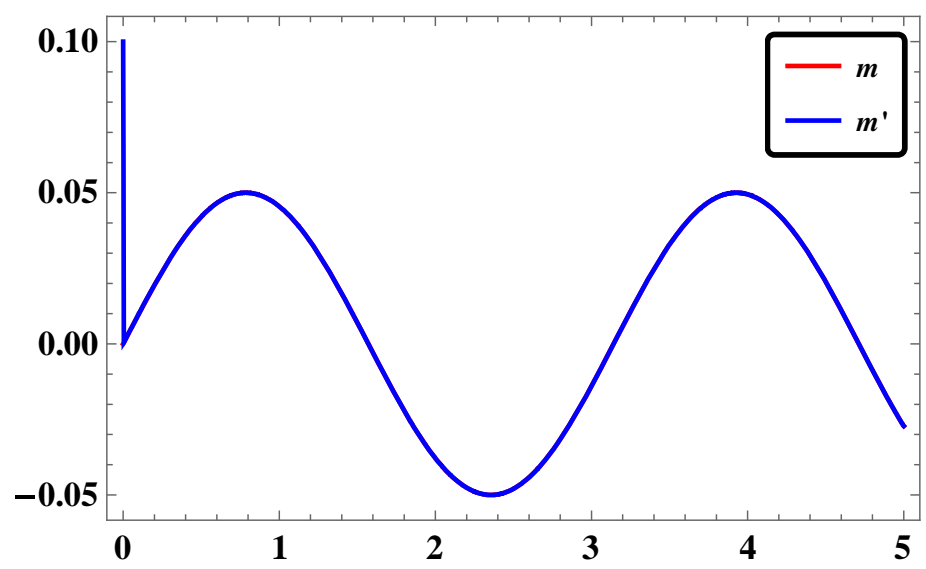

Figure 31. Time Series of $m \& m^{\prime}$. 


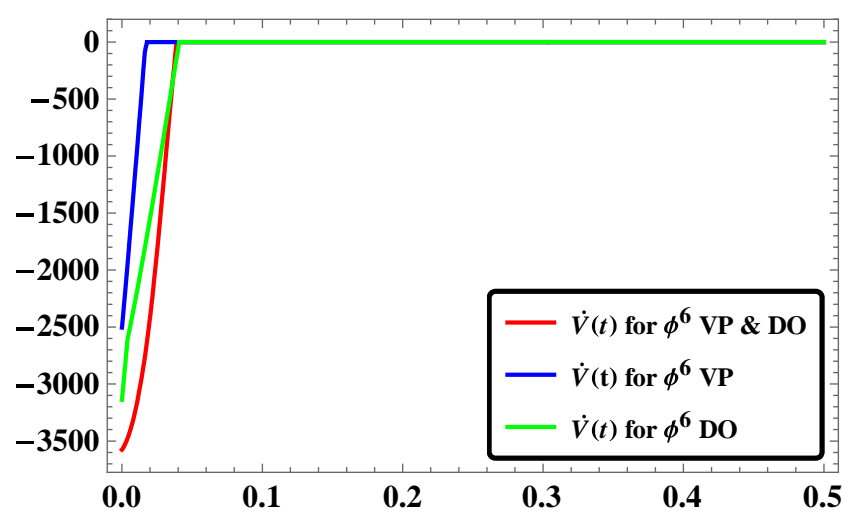

Figure 32. Time Series of $\dot{V}(t)$ in all cases.

\subsection{Numerical Simulations and Discussion}

The main aim of Section 3 was to implement the RASMC on the two identical pairs of $\Phi^{6}$-VDPO and DO and one non-identical pair of $\Phi^{6}-\mathrm{VDPO}$ and DO for synchronization purpose. It has been observed that not only RASMC is found to be very effective for all the three pairs under consideration; it is also effective for a secure communication scheme. To the best of our knowledge, this has been done for the first time using RASMC. The time of stabilization of synchronization is very short (nearly $t=0.2$ ) for all the cases that can be observed in the plotted time series (Figures 14-32). We can say now that the time response of stabilization of synchronization is much quicker in RASMC technique than it was in LAC.

\section{Conclusions}

In this computational cum comparative study, the problem of chaotic synchronization of chaotic systems is repeated using Mathematica via LAC technique. It has been found that the time response of stabilization of synchronization is reduced by half when it is done using Mathematica. On the other hand, when the same pairs are synchronized via RAMSC, the time response of stabilization of synchronization is found to be much faster than the LAC technique. Finally, we conclude the following remarkable features of our proposed study:

(1) The time response of stabilization of synchronization for LAC in our study was found to occur with rapid convergence if simulation is done with Mathematica.

(2) For the same pairs of master and slave systems considered in our study, the RASMC is found to be more effective in terms of time response of stabilization of synchronization.

On the basis of these two points, we conclude that selection of appropriate mathematical tools for simulation and technique for synchronization is very important.

Acknowledgments: The authors would like to express their gratitude to the honorable reviewers who suggested many worthwhile changes to improve the work of this manuscript.

Author Contributions: Azizan Bin Saaban performed the literature review and proposed the problem. Adyda Binti Ibrahin and Israr Ahmad performed the analytical analysis and wrote the paper. Mohammad Shahzad performed the numerical simulations and revised the manuscript.

Conflicts of Interest: The authors declare no conflict of interest.

\section{References}

1. Shahzad, M.; Pham, V.T.; Ahmad, M.A.; Jafari, S.; Hadaeghi, F. Synchronization and circuit design of a chaotic system with coexisting hidden attractors. Eur. Phys. J. Spec. Top. 2015, 224, 1637-1652. [CrossRef]

2. Yao, L.; Cai, G. Chaos Synchronization of a New Hyperchaotic Finance System Via a Novel Chatter Free Sliding Mode Control Strategy. Int. J. Nonlinear Sci. 2014, 17, 176-181. 
3. Mobayen, S. A Novel Global Sliding Mode Control Based on Exponential Reaching Law for a Class of under Actuated Systems with External Disturbances. J. Comput. Nonlinear Dyn. 2015, 11, 021011-021019. [CrossRef]

4. Azar, A.T.; Zhu, Q. Advances and Applications in Sliding Mode Control Systems; Springer International Publishing: Cham, Switzerland, 2014.

5. Shahzad, M.; Raziuddin, M. Synchronization Of Three Dimensional Cancer Model With Rosslar System Using A Robust Adaptive Sliding Mode Controller. Int. J. Math. Arch. 2015, 6, 123-132.

6. Shahzad, M.; Saaban, A.B.; Ibrahim, A.B.; Ahmad, I. Adaptive control to synchronise and anti-synchronise two identical time delay Bhalekar-Gejji chaotic systems with unknown parameters. Int. J. Autom. Control 2015, 9, 211-227. [CrossRef]

7. Ma, J.; Zhang, A.; Xia, Y.; Zhang, L. Optimize design of adaptive synchronization controllers and parameter observers in different hyperchaotic systems. Appl. Math. Comput. 2010, 215, 3318-3326. [CrossRef]

8. Lin, C.; Peng, Y.; Lin, M. CMAC-based adaptive backstepping synchronization of uncertain chaotic systems. Chaos Soliton Fract. 2009, 42, 981-988. [CrossRef]

9. Bai, E.W.; Lonngren, K.E. Synchronization of two Lorenz system using Active control. Phys. Rev. Lett. 1997, 64, 1199-1196. [CrossRef]

10. Vincent, U.E. Synchronization of Identical and Nonidentical 4-D Chaotic Systems using Active Control. Chaos Solitons Fract. 2008, 37, 1065-1075. [CrossRef]

11. Njah, A.N. Synchronization and Anti-synchronization of double hump Duffing-Van der Pol Oscillators via Active Control. J. Inf. Comput. Sci. 2009, 4, 243-250.

12. Njah, A.N. Synchronization via active control of parametrically and externally excited $\Phi^{6}$ —Van der Pol and duffing oscillators and application to secure communications. J. Vib. Cont. 2011, 17, 493-504. [CrossRef]

13. Shahzad, M.; Ahmad, I. Experimental study of synchronization \& Anti-synchronization for spin orbit problem of Enceladus. Int. J. of Cont. Sci. Eng. 2013, 3, 41-47.

14. Pisarchik, A.N.; Arecchi, F.T.; Meucci, R.; Garbo, A.D. Synchronization of Shilnikov Chaos in $\mathrm{CO}_{2} \mathrm{Laser}_{\text {with }}$ Feedback. Laser Phys. 2014, 11, 1235-1239.

15. Hammami, S. State feedback-based secure image cryptosystem hyperchaotic synchronization. ISA Trans. 2015. [CrossRef] [PubMed]

16. Rafikov, M.; Balthazar, J.M. On control and synchronization in chaotic and hyperchaotic systems via linear feedback control. Commun. Nonlinear Sci. Numer. Simul. 2008, 13, 1246-1255. [CrossRef]

17. Luo, R.Z.; Zhang, F. Finite-Time Modified Projective Synchronization of Unknown Rossler and Coullet Systems. Commun. Control Sci. Eng. 2013, 1, 51-56.

18. Cai, N.; Jing, Y.; Zhang, S. Modified projective synchronization of chaotic systems with disturbances via active sliding mode control. Commun. Nonlinear Sci. Numer. Simul. 2010, 15, 1613-1620. [CrossRef]

19. Fu, G. Robust adaptive modified function projective synchronization of different hyperchaotic systems subject to external disturbance. Commun. Nonlinear Sci. Numer. Simul. 2012, 17, 2602-2608. [CrossRef]

20. Ahmad, I.; Saaban, A.; Ibrahim, A.; Shahzad, M. A Research on the Synchronization of Two Novel Chaotic Systems Based on a Nonlinear Active Control Algorithm. Eng. Tech. Appl. Sci. Res. 2015, 5, 739-747.

21. Singh, P.P.; Singh, J.P.; Roy, B.K. Synchronization and anti-synchronization of Lu and bhalekar-Gejji chaotic systems using nonlinear active control. Chaos Solitons Fract. 2014, 69, 31-39. [CrossRef]

22. Ahmad, I.; Saaban, A.B.; Ibrahim, A.B.; Shahzad, M. On Globally Exponential Stable Complete Synchronization of Nearly Identical Hyperchaotic Systems via Linear Active Control. Ciênc. Téc. Vitiviníc. Sci. Technol. J. 2015, 30, 141-154.

23. Shahzad, M. The Improved Results with Mathematica and Effects of External Uncertainty \& Disturbances on Synchronization using a Robust Adaptive Sliding Mode Controller: A Comparative Study. Nonlinear Dyn. 2015, 79, 2037-2054.

24. Pourmahmood, M.; Khanmohammadi, S.; Alizadeh, G. Synchronization of two different uncertain chaotic systems with unknown parameters using a robust adaptive sliding mode controller. Commun. Nonlinear Sci. Numer. Simul. 2011, 16, 2853-2868. [CrossRef]

25. Vaidyanathan, S.; Rasappan, S. Global Chaos Synchronization for WINDMI and Coullet Chaotic Systems Using Active Control. J. Cont. Eng. Tech. 2013, 3, 69-75.

26. Ahmad, I.; Saaban, A.B.; Ibrahim, A.B.; Shahzad, M. A Research on Active Control to Synchronize a New 3D Chaotic System. Systems 2016, 4. [CrossRef] 
27. Ahmad, I.; Saaban, A.B.; Ibrahim, A.B.; Shahzad, M.; Naveed, N. The Synchronization of Chaotic Systems with Different Dimensions by a Robust Generalized Active Control. Int. J. Light Electron. Opt. 2016, 127, 4859-4871. [CrossRef]

28. Ahmad, I.; Saaban, A.; Ibrahim, A.; Shahzad, M. Global Chaos Identical and Nonidentical Synchronization of a New Chaotic System Using Linear Active Control. Complexity 2014. [CrossRef]

29. Siewe, S.M.; Moukam, K.F.M.; Tchawoua, C.; Woafo, P. Bifurcation and chaos in triple-well $\Phi^{6} \_$Van der Pol oscillator driven by external and parametric excitations. Phys. A 2005, 357, 383-396. [CrossRef]

30. Tchoukuegno, R.; Nbendjo, B.R.N.; Woafo, P. Resonant oscillations and fractal basin boundaries of a particle in a $\Phi^{6}$ potential. Phys. A 2002, 304, 362-368. [CrossRef]

31. Tchoukuegno, R.; Nbendjo, B.R.N.; Woafo, P. Linear feedback and parametric controls of vibrations and chaotic escape in a $\Phi^{6}$ potential. Int. J. Nonlinear Mech. 2003, 38, 531-541. [CrossRef]

32. Li, W.; Chang, K. Robust synchronization of drive-response chaotic systems via adaptive sliding mode control. Chaos Soliton Fract. 2009, 39, 2086-2092. [CrossRef]

33. Yan, J.; Hung, M.; Chiang, T.; Yang, Y. Robust synchronization of chaotic systems via adaptive sliding mode control. Phys. Lett. A 2006, 356, 220-225. [CrossRef]

34. Aghababa, M.P.; Heydari, A. Chaos synchronization between two different chaotic systems with uncertainties, external disturbances, unknown parameters and input nonlinearities. Appl. Math. Model. 2012, 36, 1639-1652. [CrossRef]

35. Aghababa, M.P.; Akbari, M.E. A chattering-free robust adaptive sliding mode controller for synchronization of two different chaotic systems with unknown uncertainties and external disturbances. Appl. Math. Comput. 2012, 218, 5757-5768. [CrossRef]

36. Khan, A.; Shahzad, M. Synchronization of circular restricted three body problem with Lorenz hyper chaotic system using a robust adaptive sliding mode controller. Complexity 2013, 18, 58-64. [CrossRef]

(C) 2016 by the authors; licensee MDPI, Basel, Switzerland. This article is an open access article distributed under the terms and conditions of the Creative Commons Attribution (CC-BY) license (http://creativecommons.org/licenses/by/4.0/). 